\title{
Implantable photonic neural probes for light-sheet fluorescence brain imaging
}

\author{
Wesley D. Sacher, ${ }^{\text {a,b,c,d, }} *$ Fu-Der Chen, ${ }^{c, \dagger}$ Homeira Moradi-Chameh, ${ }^{\text {e, }}{ }^{\dagger}$

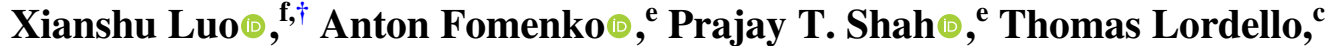 \\ Xinyu Liu, ${ }^{a}$ Ilan Felts Almog, ${ }^{c}$ John N. Straguzzi, ${ }^{d}$ Trevor M. Fowler, \\ Youngho Jung, ${ }^{\text {c,d }}$ Ting Hu, ${ }^{\text {g Junho Jeong, }}$ Andres M. Lozano ${ }^{\text {e,h }}$ \\ Patrick Guo-Qiang Lo, ${ }^{\mathrm{f}}$ Taufik A. Valiante, ${ }^{\mathrm{c}, \mathrm{e}, \mathrm{h}, \mathrm{i}}$ Laurent C. Moreaux, ${ }^{\mathrm{a}}$ \\ Joyce K. S. Poon ${ }^{\text {, }, d}$ and Michael L. Roukes ${ }^{\text {a,b, }}{ }^{*}$ \\ ${ }^{a}$ California Institute of Technology, Division of Physics, Mathematics, and Astronomy, \\ Pasadena, California, United States \\ ${ }^{b}$ Kavli Nanoscience Institute, California Institute of Technology, Pasadena, California, \\ United States \\ 'University of Toronto, Department of Electrical and Computer Engineering, Toronto, \\ Ontario, Canada \\ ${ }^{\mathrm{d}}$ Max Planck Institute of Microstructure Physics, Halle, Germany \\ ${ }^{e}$ University Health Network, Krembil Research Institute, Division of Clinical and \\ Computational Neuroscience, Toronto, Ontario, Canada \\ ${ }^{\mathrm{f}}$ Advanced Micro Foundry Pte. Ltd., Singapore \\ ${ }^{g}$ Agency for Science Technology and Research (A*STAR), Institute of Microelectronics, \\ Singapore \\ ${ }^{\text {h}}$ University of Toronto, Toronto Western Hospital, Division of Neurosurgery, \\ Department of Surgery, Toronto, Ontario, Canada \\ ${ }^{i}$ University of Toronto, Institute of Biomaterials and Biomedical Engineering, Toronto, \\ Ontario, Canada
}

\begin{abstract}
Significance: Light-sheet fluorescence microscopy (LSFM) is a powerful technique for highspeed volumetric functional imaging. However, in typical light-sheet microscopes, the illumination and collection optics impose significant constraints upon the imaging of non-transparent brain tissues. We demonstrate that these constraints can be surmounted using a new class of implantable photonic neural probes.

Aim: Mass manufacturable, silicon-based light-sheet photonic neural probes can generate planar patterned illumination at arbitrary depths in brain tissues without any additional micro-optic components.
\end{abstract}

Approach: We develop implantable photonic neural probes that generate light sheets in tissue. The probes were fabricated in a photonics foundry on 200-mm-diameter silicon wafers. The light sheets were characterized in fluorescein and in free space. The probe-enabled imaging approach was tested in fixed, in vitro, and in vivo mouse brain tissues. Imaging tests were also performed using fluorescent beads suspended in agarose.

Results: The probes had 5 to 10 addressable sheets and average sheet thicknesses $<16 \mu \mathrm{m}$ for propagation distances up to $300 \mu \mathrm{m}$ in free space. Imaging areas were as large as $\approx 240 \mu \mathrm{m} \times$ $490 \mu \mathrm{m}$ in brain tissue. Image contrast was enhanced relative to epifluorescence microscopy.

Conclusions: The neural probes can lead to new variants of LSFM for deep brain imaging and experiments in freely moving animals.

(C) The Authors. Published by SPIE under a Creative Commons Attribution 4.0 Unported License. Distribution or reproduction of this work in whole or in part requires full attribution of the original publication, including its DOI. [DOI: 10.1117/1.NPh.8.2.025003]

\footnotetext{
*Address all correspondence to Wesley D. Sacher, wesley.sacher@mpi-halle.mpg.de; Michael L. Roukes, roukes@caltech.edu ${ }^{\dagger}$ Equal contribution
} 
Keywords: neurophotonics; integrated optics; functional imaging; microscopy; biophotonics; light-sheet fluorescence microscopy.

Paper 20060R received Aug. 12, 2020; accepted for publication Mar. 4, 2021; published online Apr. 19, 2021.

\section{Introduction}

New methods in optogenetics ${ }^{1-3}$ and, especially, the advent of fluorescent reporters of neuronal activity, have opened many novel approaches for actuating and recording neural activity en masse, through the use of powerful free-space single-photon and multi-photon microscopy methods. ${ }^{4-8}$ However, existing approaches to functional imaging of the brain have significant limitations. Single-photon (1P) epifluorescence imaging readily lends itself to high frame-rate wide-field microscopy, but, in its simplest implementations, image contrast is hampered by out-of-focus background fluorescence, and the depth of imaging is restricted by the optical attenuation in the tissue. Confocal imaging improves the contrast by optical sectioning, and out-of-focus light is rejected using a pinhole; however, a laser beam must be scanned across each point of the tissue and this significantly slows the image acquisition rate. ${ }^{9}$ Multi-photon microscopy is also inherently a point or line scanning method, but because it uses infrared excitation (which provides a longer optical attenuation length ${ }^{5}$ ), the imaging depth in brain tissue can be extended to $\sim 1 \mathrm{~mm}$ and the focus of the light beam can be rastered in three-dimensions to achieve volumetric imaging. ${ }^{5,10-12}$

Light-sheet fluorescence microscopy (LSFM), which is also known as selective-plane illumination microscopy, combines the benefits of fast wide-field imaging, volumetric imaging, and optical sectioning. ${ }^{13}$ In conventional LSFM, a thin sheet of excitation light is generated either by cylindrical focusing elements or digitally scanning a Gaussian or Bessel beam. ${ }^{14-16}$ The sheet is translated in one dimension across the sample; the fluorescence images are then sequentially collected in the direction perpendicular to the illumination plane to form a volumetric image. ${ }^{17}$ With digitally scanned two-photon (2P) LSFM, it is also possible to increase the optical penetration depth. ${ }^{16}$ Non-digitally scanned 1P-LSFM is inherently faster than point- or line-scan methods; and since the illumination is restricted to a plane, photobleaching, phototoxicity, and out-of-focus background fluorescence are reduced compared to epifluorescence microscopy. However, conventional LSFM requires two orthogonal objective lenses, and appropriately positioning these largely limits the imaging modality to quasi-transparent organisms (e.g., C. elegans, Drosophila embryos, and larval zebrafish), chemically cleared mammalian brains, ${ }^{17}$ and brain slices. ${ }^{18}$ An LSFM variant called swept confocally aligned planar excitation (SCAPE) microscopy, which requires only a single objective, removes these constraints. ${ }^{6,19}$ While in vivo calcium neural imaging has been demonstrated using SCAPE in mice, ${ }^{6}$ miniaturization of the system to be compatible with freely moving animal experiments remains challenging due to the additional optics required.

To make LSFM compatible with non-transparent tissues such as mammalian brains and, eventually, behavioral experiments with freely moving animals necessitates drastic miniaturization of the light-sheet generation and fluorescence imaging compared to today's archetypical table-top systems. The feasibility of fluorescence microscopy in small and lightweight form factors has already been established by way of head-mounted microscopes for 1P and 2P calcium imaging in mice, ${ }^{4,20-23}$ though the endoscopic implantation of the requisite gradient index (GRIN) lenses, with typical diameters of 0.5 to $2 \mathrm{~mm}$, displaces a significant amount of brain tissue.

On the other hand, it remains a formidable and unsolved challenge to generate light sheets by implantable elements at arbitrary brain depths, while minimizing tissue displacement and remaining compatible with a sheet-normal imaging system. For example, in Ref. 24, to generate a light sheet perpendicular to the imaging GRIN lens required implantation of a millimeter-scale prism coupled to a second GRIN lens. In another example, in Ref. 25, a single light sheet was produced from a microchip using a grating coupler (GC), a glass spacer block, and a metallic slit lens. The overall device was $>100-\mu \mathrm{m}$ thick and $>600-\mu \mathrm{m}$ wide, which would displace a significant amount of tissue upon implantation. 


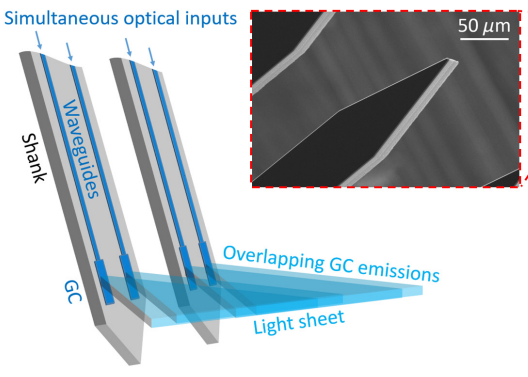

(a)

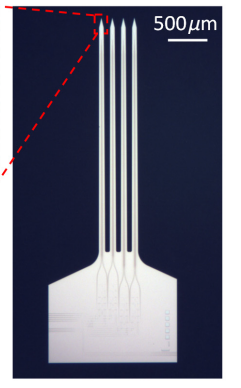

(b)

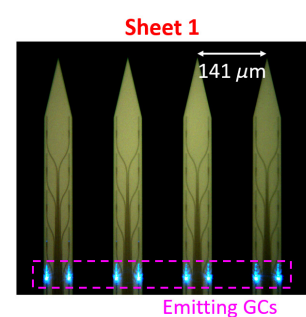

Sheet 1

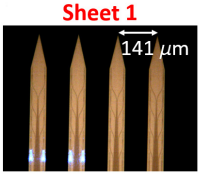

Sheet 6

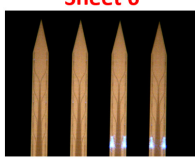

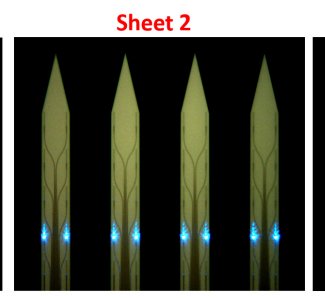

(d)
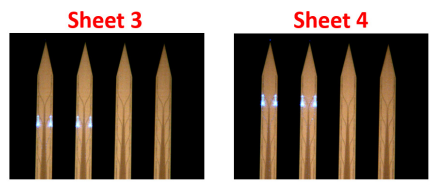

Sheet 9

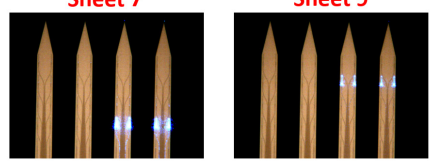

(e)

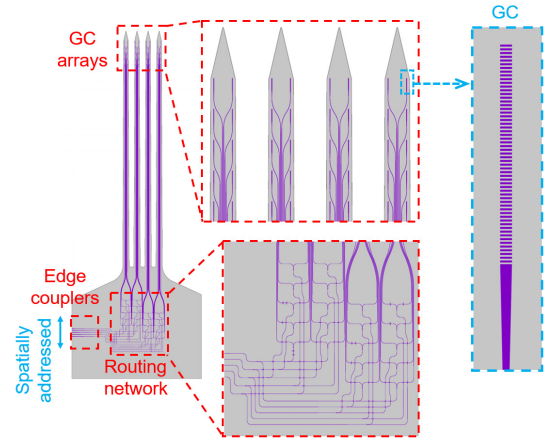

(c)

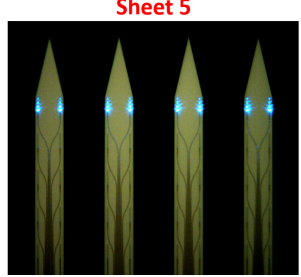

Sheet 5

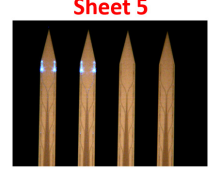

Sheet 10

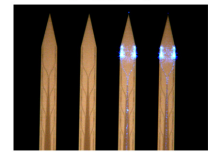

$\stackrel{\ln }{\rightarrow}$

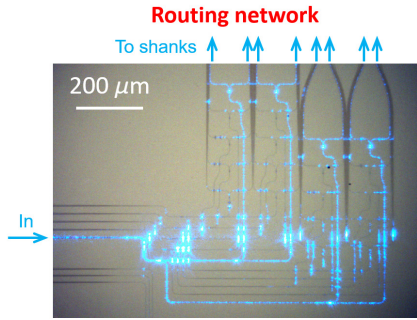

To shanks $\uparrow \uparrow \uparrow \uparrow \uparrow \uparrow \uparrow$

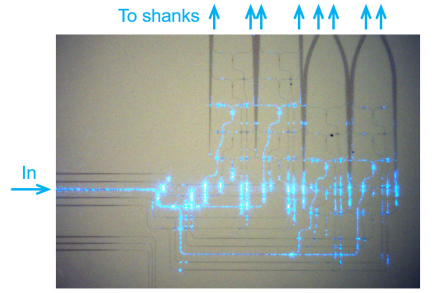

(f)

Fig. 1 Light-sheet photonic neural probes. (a) Illustration of the light-sheet synthesis method (adapted from Ref. 26). A series of simultaneously fed optical waveguides emits light via a row of GCs designed for large divergences along the sheet-axis and small divergences along the GC-axis. (b) Optical micrograph of a fabricated neural probe, (inset) scanning electron micrograph of the tip of a shank. (c) Top-down schematics of the neural probe. (d) and (e) Annotated optical micrographs of two neural probes with various GC rows emitting light sheets. (d) Neural probe design with sheets generated from four shanks. (e) Probe design with sheets generated from two shanks ("half-sheet design"). (f) Optical micrographs showing the routing network from the probe in (d) guiding light for optical inputs to two different edge couplers. The images in (d) and (e) have been contrast- and brightness-adjusted to enhance the visibility of the waveguides.

Here, we solve these challenges using wafer-scale nanophotonic technology to realize implantable, silicon-based, light-sheet photonic neural probes that require no additional microoptics. They are fully compatible with free-space fluorescence imaging (light collection) outside the brain, where the axis of collection is oriented perpendicular to the light sheets. These silicon (Si) probes synthesize light sheets in tissue using sets of nanophotonic GCs integrated onto thin, implantable, 3-mm-long Si shanks with 50 to $92 \mu \mathrm{m}$ thickness, widths that taper from 82 to $60 \mu \mathrm{m}$ along their length, and sharp tips at the distal ends. These prototype photonic neural probes (Fig. 1) are capable of generating and sequentially addressing up to five illumination planes with a pitch of $\approx 70 \mu \mathrm{m}$. Additionally, the form factor and illumination geometry of the probes open an avenue toward their integration with GRIN lens endoscopes and miniature microscopes, as shown conceptually in Fig. 2(b); offering a singular pathway to rapid, optically sectioned functional imaging at arbitrary depths in the brain.

The probes were fabricated on 200-mm Si wafers in a Si photonics foundry for manufacturing scalability and mass producibility. Elsewhere, we have used this technology to realize photonic neural probes that emit dynamically reconfigurable, patterned light with cellular-scale beam widths ${ }^{27}$ and steerable beams without moving parts, ${ }^{28}$ adding to a growing number of photonic 


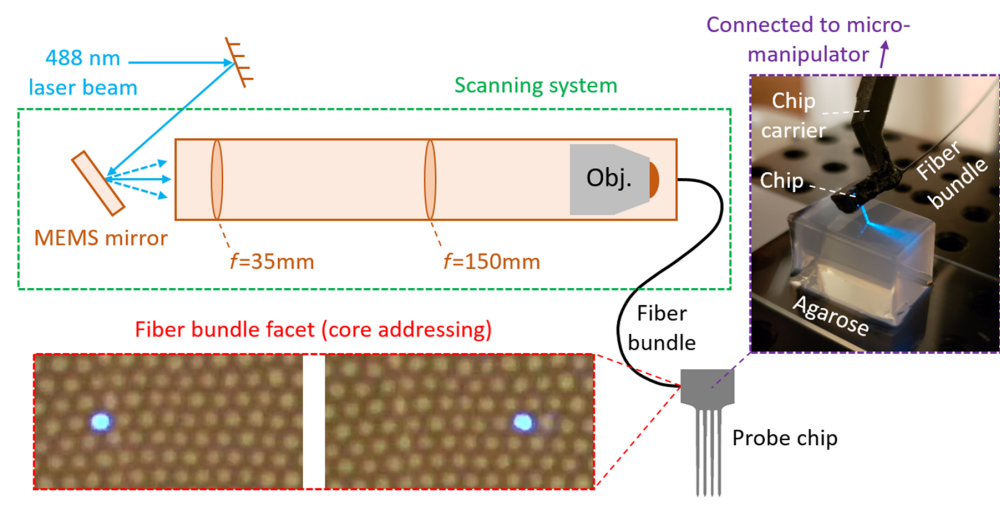

(a)

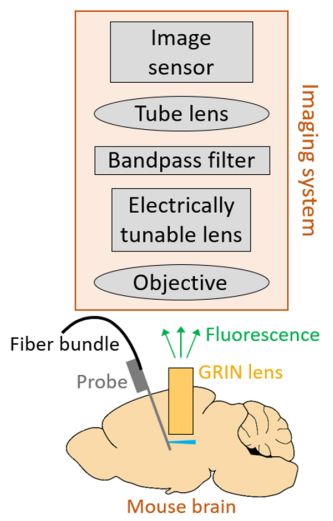

(b)

Fig. 2 Optical addressing method and proposal for deep-brain photonic-probe-enabled LSFM. (a) Schematic of the optical addressing method (not to scale). The scanning system addresses on-chip edge couplers via spatial addressing of the cores of an image fiber bundle. Bottom inset: micrographs of the distal facet of a fiber bundle connected to the scanning system with different cores addressed (adapted from Ref. 26). Top inset: annotated photograph of a packaged lightsheet neural probe inserted into an agarose block. (b) Illustration of the proposed use of the lightsheet neural probe with a GRIN lens endoscope for deep brain LSFM (not to scale). In this first investigation of the probe functionality, the configuration in (b) has not been demonstrated, and instead, the results here focus on a simpler imaging configuration where light-sheet probe illuminated samples are directly imaged with a fluorescence microscope without a GRIN lens (see Sec. 2).

neural probe demonstrations with increasing levels of integration and sophistication. ${ }^{29-31}$ In this work, we employ this integrated nanophotonics technology to realize implantable, microscale probes that form light sheets for imaging over areas as large as $\approx 240 \mu \mathrm{m} \times 490 \mu \mathrm{m}$ in brain tissue. Our preliminary results were reported in Ref. 26. Here, we report in detail the imaging properties of the light-sheet neural probes, characterizing their performance by means of suspended fluorescent beads in phantoms as well as in adult mouse brain slices. We also report a first demonstration of in vivo calcium imaging using a prototype light-sheet neural probe that we fabricated on a $100-\mathrm{mm}$ wafer, as in Ref. 27 , prior to our foundry-fabricated probes.

\section{Results}

\subsection{Photonic Neural Probes on 200-mm Silicon Wafers}

To ensure that fabrication of our photonic neural probes can be scaled up for dissemination to the neuroscience community, we have adapted from the outset foundry Si photonics manufacturing processes. The neural probes described herein were fabricated in a 200-mm Si photonic line; silicon nitride ( $\mathrm{SiN}$ ) waveguides (135-nm nominal thickness) with $\mathrm{SiO}_{2}$ cladding were patterned onto $\mathrm{Si}$ wafers, deep trenches were etched in the wafers to define the probe shapes, and the wafers were thinned to thicknesses of 50 to $92 \mu \mathrm{m}$. The shank thickness can be reduced in future iterations to $18 \mu \mathrm{m}$, as in Refs. 26 and 27. The fabrication is more fully detailed in Sec. 4, "Methods."

The light-sheet neural probe design is shown in Figs. 1(a)-1(c). Light is coupled onto the probe chip using fiber-to-chip edge couplers that taper from $5.2 \mu \mathrm{m}$ in width at the chip facet to single-mode waveguides with widths of 270 to $330 \mathrm{~nm}$. The waveguide-coupled optical power is divided between four to eight waveguides using a routing network consisting of $1 \times 2$ multimode interference splitters ${ }^{32}$ and in-plane waveguide crossings. ${ }^{33}$ The light is then guided along the implantable shanks via $1-\mu \mathrm{m}$ wide, multimode waveguides, and subsequently emitted near the distal end of the probe by a row of GCs. Light sheets are synthesized by overlapping the emission from an array of simultaneously fed GCs. Each row of GCs generates a separate light sheet. The width, period, and duty cycle of the GCs are designed to achieve a large output divergence angle 
Table 1 Light-sheet photonic neural probes described in this work.

\begin{tabular}{lccccc}
\hline \hline Probe name & $\begin{array}{c}\text { Nominal probe } \\
\text { thickness }(\mu \mathrm{m})\end{array}$ & $\begin{array}{c}\text { Shanks } \\
\text { per sheet }\end{array}$ & $\begin{array}{c}\text { Number of } \\
\text { sheets }\end{array}$ & $\begin{array}{c}\text { Emission angle } \\
\text { in fluorescein }\left(^{\circ}\right)\end{array}$ & $\begin{array}{c}\text { Sheet pitch in } \\
\text { fluorescein }(\mu \mathrm{m})\end{array}$ \\
\hline Probe 1 & 92 & 4 & 5 & 23.5 & 69 \\
Probe 2 & 92 & 2 & 10 & 19.8 & 71 \\
Probe 3 & 50 & 2 & 10 & 22.3 & 69 \\
\hline \hline
\end{tabular}

along the width-axis of the sheet, and only a small divergence along the thickness-axis. Nominal lateral GC widths, periods, and duty cycles are $1.5 \mu \mathrm{m}, 440$ to $480 \mathrm{~nm}$, and $50 \%$, respectively. By contrast, our previous neural probe designs in Refs. 27 and 28 emitted low-divergence beams that are incompatible with light-sheet synthesis.

The waveguide routing network is detailed in Fig. S1 in the Supplementary Material. The photonic components were designed for a wavelength of $488 \mathrm{~nm}$ to enable excitation of common fluorophores such as green fluorescent protein (GFP) and green calcium dyes; however, these components can also be designed for green, yellow, and red wavelengths, as we have shown in Ref. 34 for excitation of other fluorophores. The probe shanks are $3 \mathrm{~mm}$ in length and separated with a $141-\mu \mathrm{m}$ pitch; the rows of GCs integrated onto the shanks, each row corresponding to a different sheet, are separated by a $75-\mu \mathrm{m}$ pitch along the shanks. The shanks taper in width from 82 to $60 \mu \mathrm{m}$ over their length and each converges to a sharp tip at its distal end.

To rapidly switch between different sheets, we used a spatial addressing approach similar to Ref. 35 and as illustrated in Fig. 2(a). An image fiber bundle was epoxied to the probe chip on a common carrier, with each edge coupler on the probe aligned to a different core of the fiber bundle. By actuating the microelectromechanical systems (MEMS) mirror, light was input to a selected core of the fiber bundle and the corresponding input waveguide for a light sheet. The light-sheet switching speed was limited to $\approx 5 \mathrm{~ms}(0.2 \mathrm{kHz})$ in the following demonstrations, a constraint arising from the MEMS mirror. Future designs will employ optimized MEMS mirrors operating in resonance mode that can yield switching frequencies $>30 \mathrm{kHz} .{ }^{36}$ Video S1 and Video S2 demonstrate rapid switching between different light sheets from packaged probes. The fiber bundle used in these first experiments did not maintain polarization, whereas the photonic circuitry was polarization dependent. Therefore, in these first probe prototypes, the fiber bundle must be held still during imaging. This limitation can be overcome in future designs with use of polarization-maintaining multicore fibers.

Table 1 summarizes three light-sheet photonic neural probes we have carefully evaluated and report upon in this article. Beam profiles for the three probes are characterized. Probe 1 is used for imaging fluorescent beads and fixed tissue, and Probe 2 is used for in vitro imaging. In the table, the "emission angle" refers to the angle of the sheet relative to the normal of the shanks. It is noteworthy that the sheets were designed to emit at an angle of $\sim 20 \mathrm{deg}$ in tissue; this permits implanting the probe next to an imaging lens such that the light sheets can be generated beneath the lens parallel to the focal plane.

Two probe designs were investigated: a first, in which each light sheet is generated by a row of eight GCs spanning four adjacent shanks (Probe 1, with five independent sheets), and a second based on a "half-sheet design," in which each sheet is generated by a row of four GCs spanning two adjacent shanks (Probes 2 and 3, with 10 independent half-sheets). In principle, the halfsheet design roughly doubles the sheet intensity for a given input optical power to the probe at the expense of a smaller sheet width. More advanced designs can achieve even larger sheet widths by distributing GCs along $>4$ shanks at the expense of: (1) a more complex routing network with higher optical losses, and (2) higher input optical powers to the probe chip to achieve a given light-sheet intensity.

\subsection{Light-Sheet Generation}

The photonic circuitry employed in these devices is designed to provide lower loss for transverse-electric (TE) polarized light. In the following characterization and imaging work, we use 


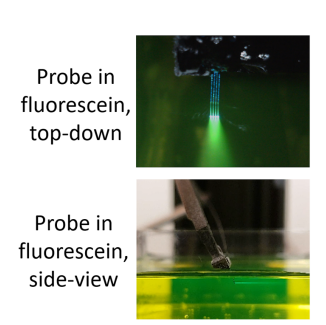

(a)

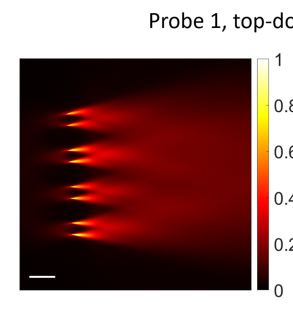

Sheet 1
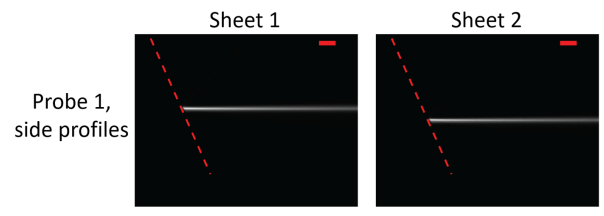

(b)

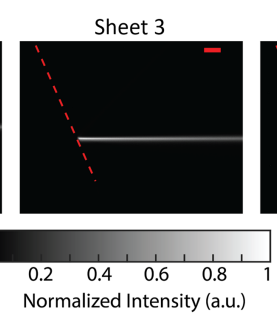

Top-down sheet profiles

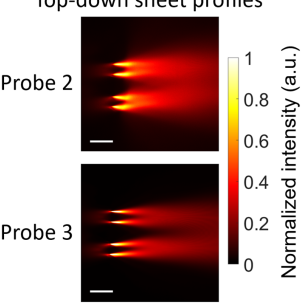

(c)

(d)

Fig. 3 Light-sheet characterization in fluorescein. (a) Top-down and side-view photographs of a light-sheet neural probe immersed in a fluorescein solution. (b) Top-down light-sheet intensity profile for Probe 1-Sheet 5 imaged with an epifluorescence microscope above the probe. The plot on the right is the sheet profile with a binned color scale to show a semi-uniform sheet region (green) over which the intensity varies by at most $2.5 \times$. (c) Top-down light-sheet intensity profiles for Probe 2-Sheet 10 and Probe 3-Sheet 7. (d) Side profile measurements of the light sheets from Probe 1 captured with a second microscope aligned to the side of the fluorescein chamber. The dashed red lines delineate the top surface of the shanks. The scale bars are $100 \mu \mathrm{m}$.

TE-polarized optical inputs to the probe chips. The insertion loss of the neural probes (defined here as the ratio of emitted power from the GCs and the input laser power to the scanning system) is summarized in the histograms for Probes 1 and 2 in Fig. S2 in the Supplementary Material. Probe 3 broke before sheet transmissions were able to be characterized.

Transmission ranged from -38 to $-20 \mathrm{~dB}$, with a median of about $-30 \mathrm{~dB}$. This large variation in transmission was due both to the irregularly positioned individual cores within the fiber bundles and alignment drift during attachment of the fiber to the probe chip. Sheet transmissions measured with a single-mode fiber with optimized alignment typically varied by $<3 \mathrm{~dB}$. In future designs, such transmission variations can be reduced by implementing optimal fiber-to-chip packaging and by employing custom multicore fibers with a constant core pitch that closely matches that of the on-chip edge couplers. Nonetheless, by modulating the input laser power while switching between sheets or adjusting the MEMS mirror positions for each sheet, these variations can be compensated with the present devices.

We have measured the intrinsic properties of light sheets generated both in free space and in non-scattering fluorescein solutions-characterizing the light-sheet thicknesses, their intensity uniformities, and the magnitudes of associated, higher-order GC diffraction. We determine the in-plane sheet intensity profile by imaging top-down while the probes are immersed in fluorescein solution [Fig. 3(a)]. When imaging from the side, the sheet thickness is overestimated since out-of-focus light can also be captured. In the free-space method, Fig. 4(a), a coverslip coated on one side with a fluorescent thin film is placed above the probe parallel to the shanks, and a crosssection of the beam profile is imaged on the coverslip. The light-sheet intensities were volumetrically profiled versus propagation distance by translating the probe relative to the coverslip.

Figures 3(b)-3(c) show top-down fluorescence light-sheet profiles from the probes in fluorescein. The GC emissions diverge and overlap to form regions of moderately uniform illumination. For Probe 1, the semi-uniform region, which we define to be the region where the maximum intensity variations were $<2.5 \times$, is the green region in the binned-color-scale sheet profile of Fig. 3(b). The semi-uniform illumination region forms a continuous sheet at a propagation distance of about $180 \mu \mathrm{m}$ and spans an area $>0.22 \mathrm{~mm}^{2}$. In scattering media such as brain tissue, the semi-uniform illumination region will form at shorter propagation distances away 


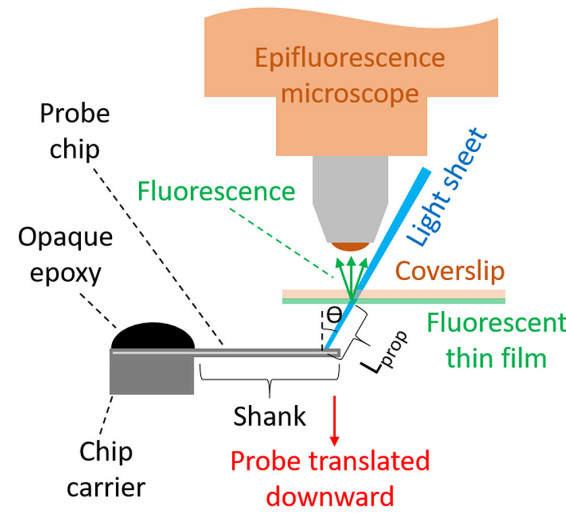

(a)

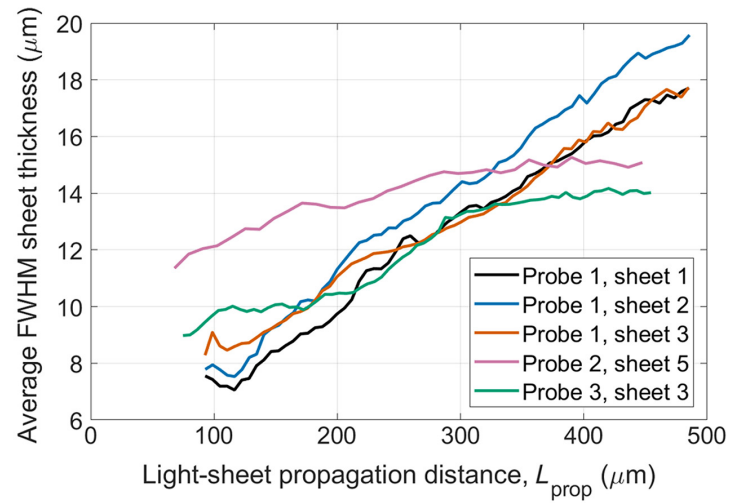

(b)
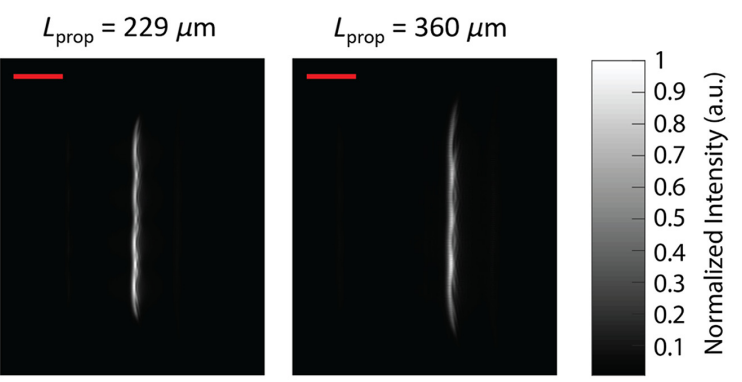

(c)

Fig. 4 Free-space light-sheet profile measurements. (a) Illustration of the light-sheet profile measurement protocol using a coverslip coated with a fluorescent thin film (not to scale). Fluorescence images of the coverslip provide cross-sectional profiles of the incident light sheet, and vertical translation of the probe enables volumetric profiling. (b) Average FWHM light-sheet thicknesses versus propagation distance for sheets from Probes 1 to 3 . The sheet thicknesses are averaged over the sheet width [vertical axis in (c)] for each sheet cross-section. (c) Light-sheet crosssections imaged at various light-sheet propagation distances, $L_{\text {prop }}$, for Probe 1 . The scale bars are $150 \mu \mathrm{m}$.

from the probe. The side profiles of the Probe 1 light sheets in fluorescein are shown in Fig. 3(d), and side profiles from Probes 2 and 3 are shown in Fig. S3 in the Supplementary Material. Weak second-order diffraction results in an additional, upward-pointing beam for each sheet; this is not visible in Fig. 3(d) due to the low second-order diffraction of Probe 1, but it is visible for Probe 2 in Fig. S3 in the Supplementary Material. The second-order diffraction profiles were similar to the light sheets, forming "second-order light sheets." The light-sheet optical powers were $>15 \times$, $3 \times$, and 16× larger than the second-order diffraction powers for Probes 1 to 3, respectively.

Figure 4(c) shows light-sheet cross-sections at several propagation distances for Probe 1 imaged with the free-space beam profiling method. The extracted average full width at half maximum (FWHM) light-sheet thickness versus propagation distance for sheets from Probes 1 to 3 are shown in Fig. 4(b). The sheet thicknesses, averaged over the width of each sheet cross-section, are $<16 \mu \mathrm{m}$ for propagation distances up to $300 \mu \mathrm{m}$. Since the coverslip was not perpendicular to the sheet propagation axis, the propagation angle of each sheet is used to convert the thickness of the sheet projection on the coverslip to a sheet thickness corrected for alignment perpendicular to the propagation axis. The apparent reduction of FWHM divergence for Probes 2 and 3 sheets at propagation distances above $300 \mu \mathrm{m}$ in Fig. 4(b) is a consequence of the evolution of the sheet shape. The full width at $1 / e^{2}$ of maximum thickness (Fig. S4 in the Supplementary Material), in general, increases linearly with propagation distance.

Small amplitude fringes are visible in the sheet cross-sections in Fig. 4(c) and the top-down profiles in Fig. 3. These fringes are caused by multipath interference from the multiple GCs that contribute to each sheet. The interference pattern is related to the differing waveguide path lengths connected to each GC and the coherence length of the laser. In brain tissue, we expect these fringes will be smoothed (suppressed) by the scattering properties of the medium. 


\subsection{Light-Sheet Fluorescence Imaging}

We investigate the efficacy of the probes for LSFM by first imaging fluorescent beads suspended in agarose, and then by imaging fixed and in vitro brain tissues. Since most miniaturized microscopes today use wide-field $1 \mathrm{P}$ fluorescence imaging, we compare the images obtained with the light-sheet probe illumination against those with epi-illumination using the same microscope. Figures 5(a)-5(c) illustrate the imaging setup. An electrically tunable lens was attached to the

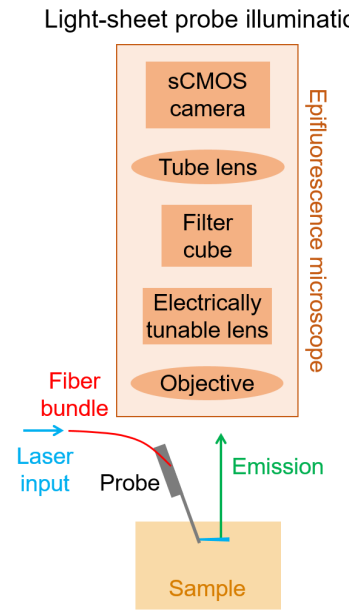

(a)

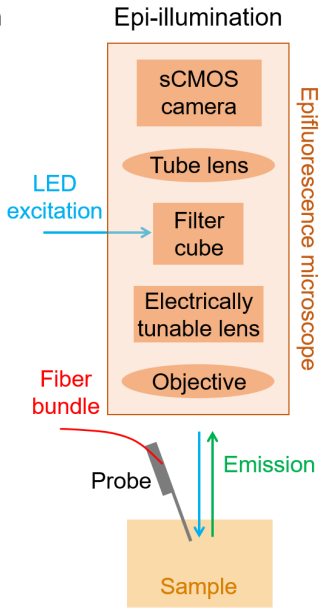

(b)

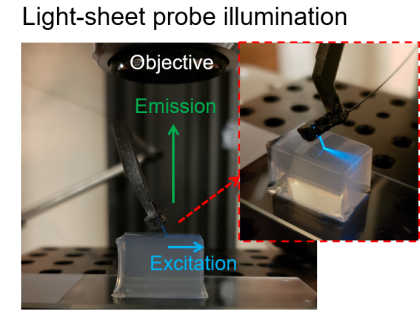

Epi-illumination

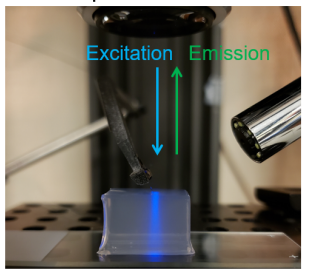

(c)

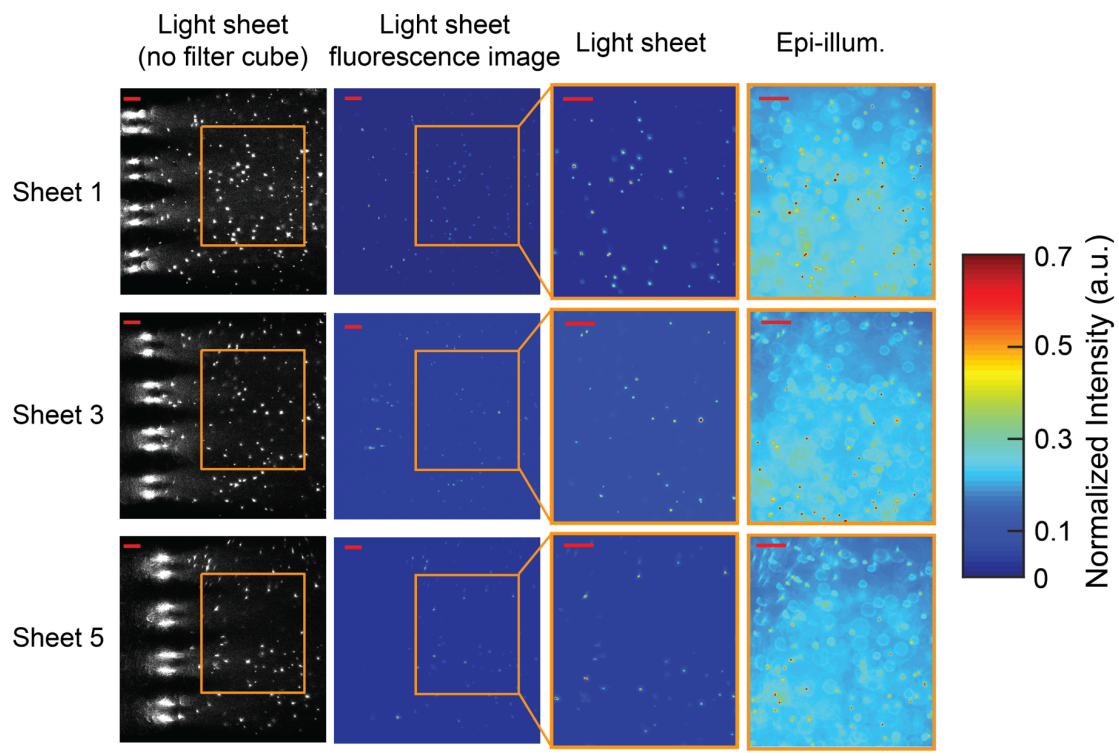

(d)

Fig. 5 Imaging of fluorescent beads suspended in agarose. Illustrations of the imaging apparatus for (a) light-sheet probe illumination and (b) microscope epi-illumination (not to scale). (c) Photographs of Probe 1 inserted into an agarose block during light-sheet probe illumination and microscope epi-illumination. (d) Imaging of fluorescent beads suspended in an agarose block using light-sheet illumination from Probe 1 (Sheets 1, 3, and 5) and epi-illumination. First column: light-sheet illumination images with no filter cube in the microscope path to show both scattered excitation light and fluorescence. The eight large bright spots at the left of the images are the emitting GCs on the shanks. Second column: fluorescence images with light-sheet illumination and the filter cube in the microscope path. Third and fourth columns: fluorescence images of the regions of interest (ROls) delineated by the orange boxes with light-sheet and epi-illumination, respectively. The epi-illumination images were captured at the same focal planes as the corresponding light-sheet images. The second to fourth columns are normalized to the maximum intensity in each image and the color scale is truncated at 0.7 to enhance bead visibility. The scale bars are $50 \mu \mathrm{m}$. 


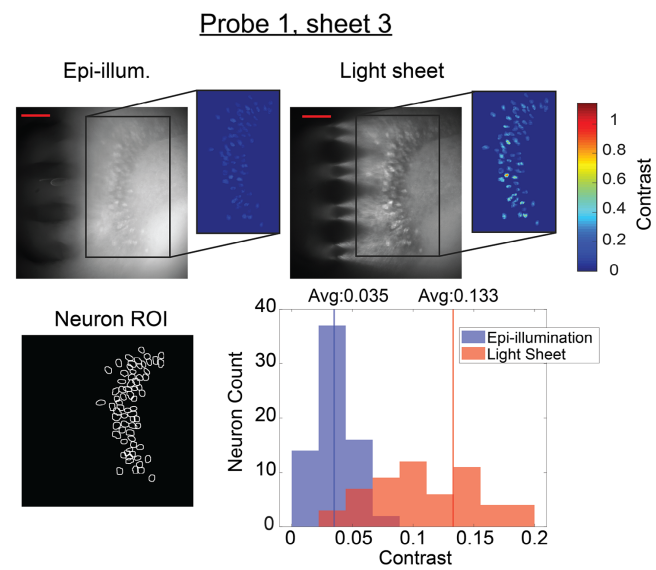

(a)

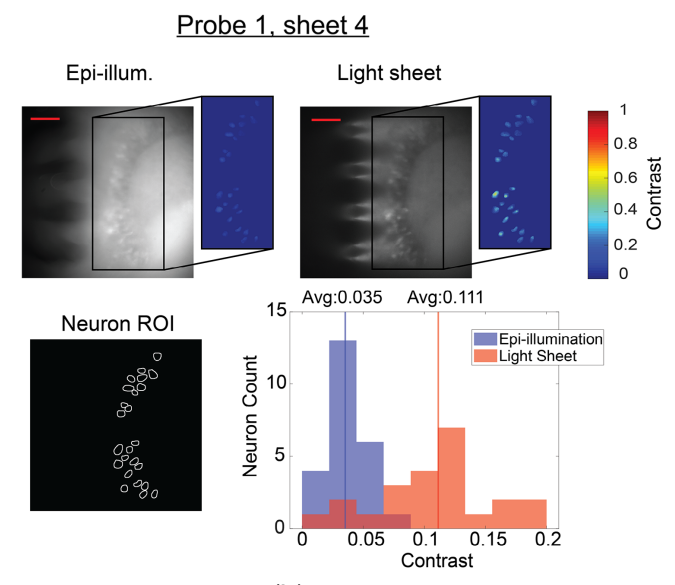

(b)

Fig. 6 Comparison of light-sheet neural probe illumination and microscope epi-illumination for fluorescence imaging of fixed brain tissue (dentate gyrus) from a Thy1-GCaMP6s mouse. Two adjacent light sheets from Probe 1 were used: (a) Sheet 3 and (b) Sheet 4 . Sheet 3 was $\approx 60 \mu \mathrm{m}$ in depth from the surface of the brain tissue, and Sheet 4 was $69 \mu \mathrm{m}$ deeper than Sheet 3. Top row: fluorescence images for epi- and light-sheet illumination with insets indicating the contrast of neurons within an ROI of high neuron density. The scale bars are $100 \mu \mathrm{m}$. Bottom row: ROls of identified neurons and corresponding histograms of image contrast for the identified neurons; the contrast of each neuron is the average over its ROI.

back of the objective to provide fast focus adjustment to the different light-sheet depth planes. When the epi-illumination was on, the input to the probe was off, and vice versa. The comparisons are performed at the same image plane, i.e., the tunable lens and microscope objective are not adjusted when switching between light-sheet and epi-illumination. The probe insertion angle was set to orient the light sheets parallel to the top surface of the sample (sheet-normal imaging).

To demonstrate optical sectioning, Probe 1 was inserted into an agarose block containing 3- $\mu$ m-diameter fluorescent beads. Figure 5(d) shows the fluorescence images captured using three of the sheets of Probe 1 compared with epifluorescence images. Significantly out-of-focus beads and fluorescence are not present with light-sheet probe illumination. This yields a dramatic reduction of the background intensity in comparison with epi-illumination. We quantify the gain in contrast in imaging experiments with tissue slices; discussed next. Video S3 shows a simple proof-of-concept volumetric imaging example. The video demonstrates fluorescence imaging of fluorescent beads in an agarose block with switching between three of the probe-generated sheets, and synchronized focus switching enabled by the electrically tunable lens.

We subsequently imaged the hippocampus in a fixed brain slice obtained from a Thy1GCaMP6s mouse. Figure 6 shows the probe- and epi-illuminated fluorescence images captured following insertion of Probe 1 into the fixed tissue. The tissue was about 1-mm thick, and imaging was performed with Sheets 3 and 4. Again, the probe-illuminated images showed remarkably less background fluorescence than epi-illumination. Neurons are observable over a sheet area of $\approx 240 \mu \mathrm{m} \times 490 \mu \mathrm{m}$ for Sheet 3, and different neurons are visible with Sheet 3 versus Sheet 4 illumination. The neurons in the image from Sheet 4, which was $69 \mu \mathrm{m}$ deeper in the tissue than Sheet 3, appear less in focus; this is due to the scattering of the fluorescence emission in the tissue.

To quantify the difference in contrast between probe- and epi-illumination, an algorithm described in Supplementary Note 1 in the Supplementary Material is applied to identify the neurons in each image, and the neurons found in both images are selected (Fig. 6 "Neuron ROI") for contrast analysis using the definition of contrast in Supplementary Note 1. Figure 6 shows the distributions of the image contrasts of the identified neurons. The contrast distributions of the two illumination methods are statistically different $(p<0.001$, two-tailed Wilcoxon signed-rank test), with the average contrast for light-sheet illumination higher than that of epi-illumination by $3.8 \times$ for Sheet 3 and $3.2 \times$ for Sheet $4.98 .6 \%$ and $100 \%$ of the neurons for Sheets 3 and 4, respectively, exhibit higher contrast using light-sheet illumination compared to epi-illumination. The color insets in Fig. 6 show the contrast of each pixel within each neuron 

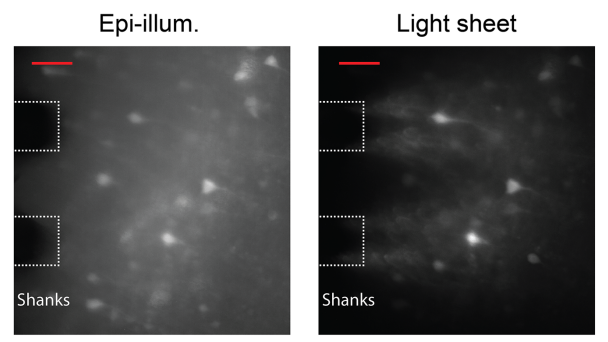

(a)

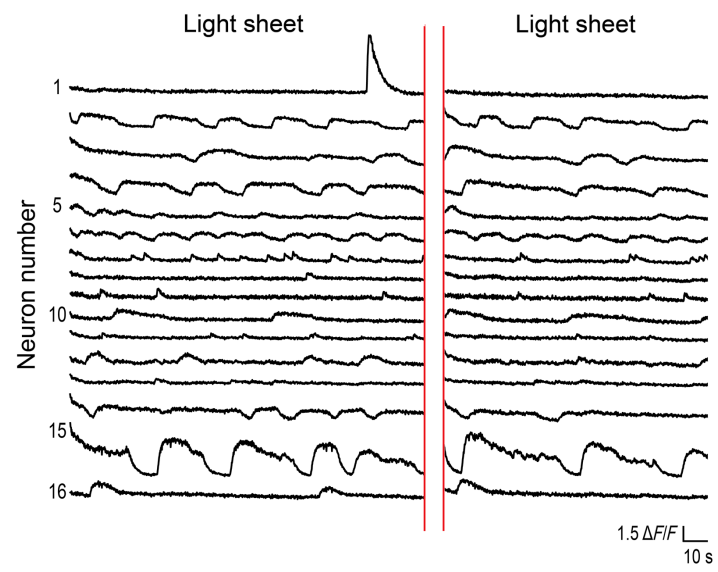

(c)

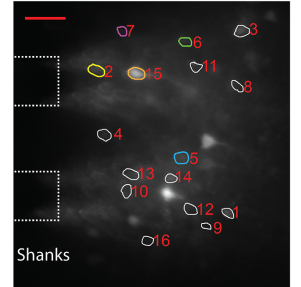

(b)

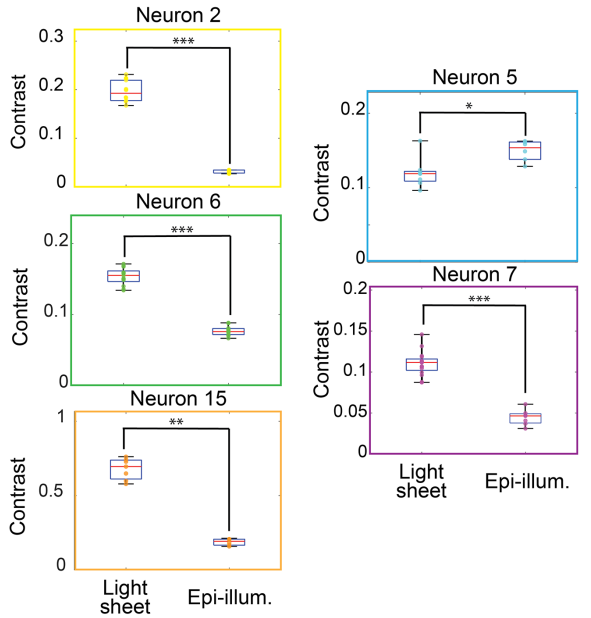

(d)

Fig. 7 In vitro functional calcium imaging of a brain slice from a Thy1-GCaMP6s mouse. (a) Maximum projection images of 142- and 92-s segments of the recorded video for light-sheet and epi-illumination, respectively, with annotations showing the approximate positions of the shanks in the image plane. The scale bars are $50 \mu \mathrm{m}$. (b) Light-sheet maximum projection image with ROls for identified active neurons shown. (c) Fluorescence change, $\Delta F / F$, time traces of all active neurons identified in (b). (d) Box plots showing the image contrast of five neurons at the peaks of all events recorded for light-sheet and epi-illumination; the numbers and colors of the box plots correspond to the ROls in (b). Asterisks indicate significant group differences. * denotes $p<0.05{ }^{* \star}$ denotes $p<0.01$ and ${ }^{\star * \star}$ denotes $p<0.001$, two-tailed Wilcoxon rank-sum test. A sample of the calcium imaging video with light-sheet probe illumination is presented (Video 1, 12.9 MB, MP4 [URL: https://doi.org/10.1117/1.NPh.8.2.025003.1]). A sample of the calcium imaging video with epi-illumination is also presented (Video 2, 29.8 MB, MP4 [URL: https://doi.org/ 10.1117/1.NPh.8.2.025003.2]). The videos are accelerated $5 \times$. The $\Delta F / F$ time traces for epiillumination are shown in Fig. 12 in Sec. 5.2.

ROI, while the histograms show neuron contrasts that are averaged over each neuron ROI. The illumination intensities for the fixed tissue and in vitro imaging are discussed in Sec. 5.1.

Photonic neural probe tests were also performed for in vitro functional calcium imaging using a 450- $\mu$ m-thick brain slice, prominently featuring the auditory cortex, from a Thy 1GCaMP6s mouse. Preparation of the tissue is described in Sec. 4, "Methods." For increased neuronal activity, the brain slice was perfused with an artificial cerebrospinal fluid (aCSF) solution containing 4-aminopyridine (4-AP). ${ }^{37}$ Figure 7 (a) shows maximum projection images over time from the probe- and epi-illumination videos of the auditory cortex region of the brain slice. Sheet 5 from Probe 2 was used, and the probe was inserted into the brain slice such that Sheet 5 was $\approx 60 \mu \mathrm{m}$ in depth from the surface of the slice. The data analysis procedure for neuron identification and extraction of the fluorescence change, $\Delta F / F$, is described in Supplementary Note 2 in the Supplementary Material. Figure 7(c) shows the $\Delta F / F$ time traces of the 16 identified active neurons using probe-illumination, and $\Delta F / F$ values as large as 5.5 were observed. Figure 7(d) shows the image contrast of five of the neurons at the peaks of all observed events; the neurons were selected with the criterion that at least five events were recorded for both light-sheet and epi-illumination. Higher image contrast is observed for light-sheet compared to epi-illumination for four of the five neurons ( $p<0.01$, two-tailed Wilcoxon rank-sum test); 
a possible explanation for the lower light-sheet contrast of the one neuron is that its depth may have been outside or on the periphery of the sheet. The ratios of the median light-sheet- and epiillumination neuron contrasts were 6.71, 0.77, 2.04, 2.46, and 3.39. Samples of calcium imaging video with both probe- and epi-illumination are shown in Video 1 and Video 2.

During calcium imaging experiments, illumination was alternated between probe- and epiillumination. The full time-traces are shown in Fig. 12 in Sec. 5.2. Due to the larger background fluorescence of epi-illumination, the apparently larger $\Delta F / F$ for light-sheet compared to epiillumination does not necessarily represent a larger signal-to-noise ratio of the calcium events. A direct comparison of signal-to-noise ratio for calcium events under these two illumination conditions is beyond the scope of this work.

To investigate the operation of the probes in tissues with a higher density of labeled neurons, tests were also performed on a green calcium dye loaded (Cal-520 AM, AAT Bioquest), $450-\mu \mathrm{m}$ thick, cerebellum brain slice from a wild type mouse. The tissue preparation is described in Sec. 4, and 4-AP was added to the aCSF perfusion solution. Probe 2 was inserted into the brain slice, and light-sheet illumination was applied from Sheet 10, which was positioned $<50 \mu \mathrm{m}$ in depth from the brain slice surface (Fig. 8). Full fluorescence time-traces with the illumination cycled between probe- and epi-illumination are shown in Fig. 13 in Sec. 5.2. Samples of the calcium imaging video are shown in Video 3 and Video S4. The labeled cells are likely a combination of neurons and glial cells. The data analysis procedure is described in Supplementary Note 2 in the Supplementary Material. For the probe illumination in Fig. 8, $\Delta F / F$ values as high as 4.3 were observed, and 73 cells were identified. The variation in maximal $\Delta F / F$ values in Fig. 8(c) may arise from a combination of the position of the cell within the sheet (both

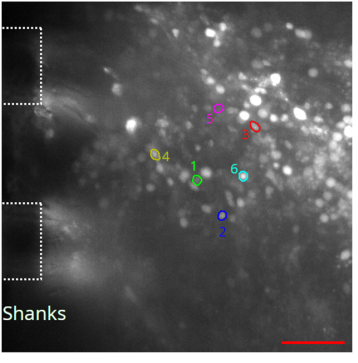

(a)

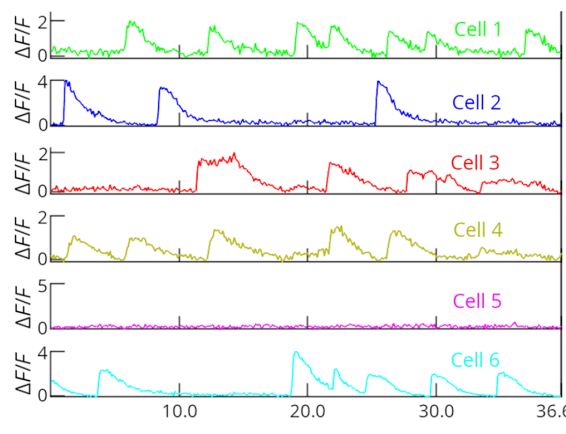

(b)

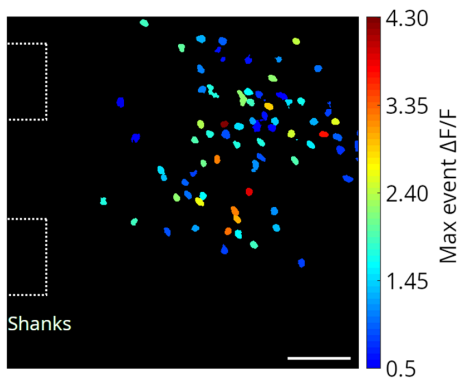

(c)

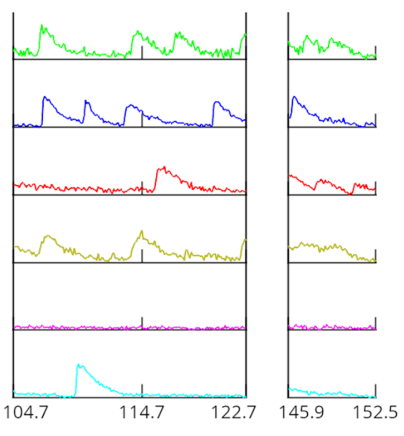

me (s)

(d)

Fig. 8 In vitro functional calcium imaging of a Cal-520 AM loaded brain slice from a wild type mouse using light-sheet neural probe illumination. (a) Maximum projection fluorescence image of $36.6 \mathrm{~s}$ of the recorded video with annotations showing the approximate positions of the shanks in the image plane. (b) Number of calcium events and (c) maximum $\Delta F / F$ observed for all identified cells; (b) and (c) are of the same scale as (a), and the scale bars are $50 \mu \mathrm{m}$. (d) Fluorescence change, $\Delta F / F$, time traces of 6 cells; the first 4 cells had the highest number of events and the last 2 had the highest peak $\Delta F / F$ among the remaining cells. The ROls for these cells are shown in (a) with colors and numbers corresponding to the time traces in (d). Breaks in the time traces correspond to times when the illumination was switched to epi-illumination. A sample of the calcium imaging video with light-sheet probe illumination is presented (Video 3, 19.6 MB, MP4 [URL: https://doi.org/10.1117/1.NPh.8.2.025003.3]). The video is real-time. 
laterally and in depth) as well as the magnitude of the calcium events. A complication in this experiment arises from the penetration depth of the dye into the slice during bath-loading; this limits the thickness of labeled tissue available to contribute to background fluorescence during epi-illumination. As a result, the image contrast enhancement of light-sheet versus epi-illumination is expected to be less than our results with Thy1-GCaMP6s mouse brain tissue (Figs. 6 and 7), where the labeling is more uniform in depth. This is confirmed by the minor contrast difference between light-sheet and epi-illumination observed for the Cal-520 AM loaded brain slice (Video 3 ,Video S4, and Fig. 13), relative to the significant contrast enhancement of lightsheet illumination in Figs. 6 and 7.

We have carried out initial in vivo tests as shown in Fig. 9 and described in Sec. 4.11. For these experiments, a light-sheet probe was inserted approximately $<200 \mu \mathrm{m}$ deep into the parietotemporal lobe of an anesthetized Thy1-GCaMP6s mouse at the approximate location of the

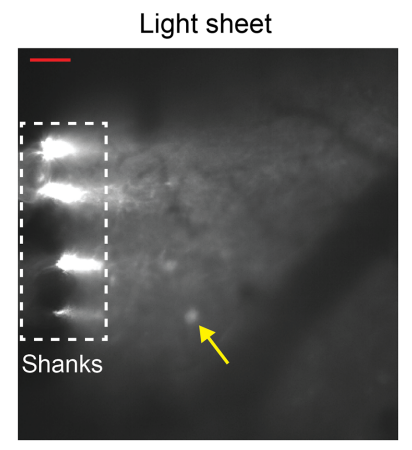

(a)

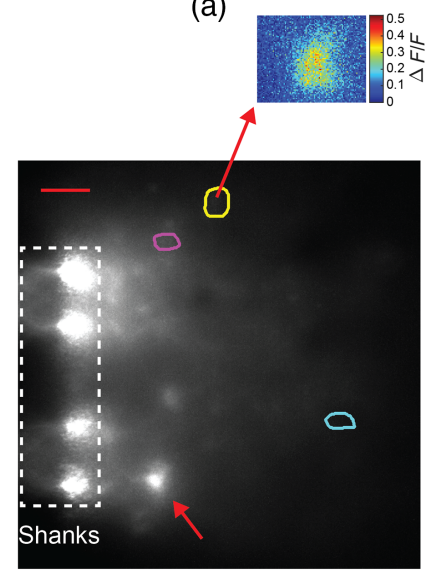

(c)
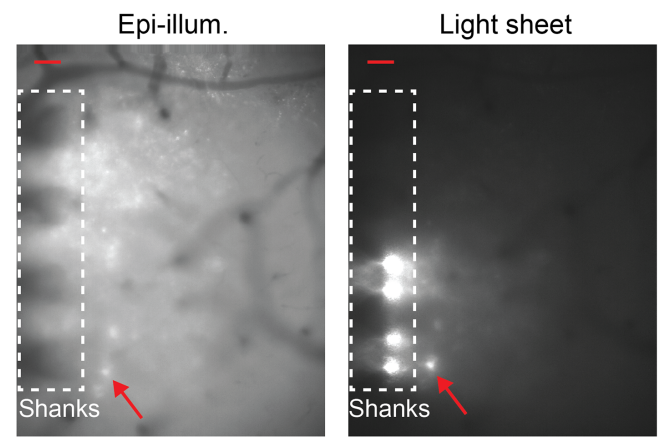

(b)
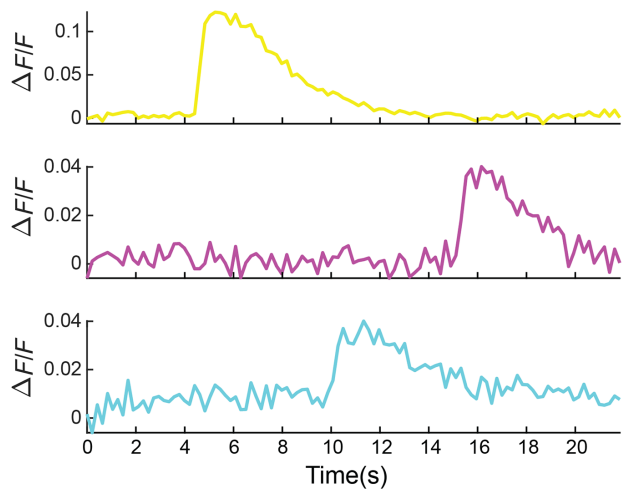

(d)

Fig. 9 In vivo fluorescence brain imaging of an anesthetized Thy1-GCaMP6s mouse. (a) Fluorescence image with light-sheet probe illumination. (b) Light-sheet and epi-illumination fluorescence images with the probe inserted into the cortex at a different position relative to (a). (c) Maximum projection fluorescence image over a segment of the video corresponding to (b) with light-sheet illumination. The image is annotated with the ROls of three neurons identified by fluorescence changes consistent with GCaMP6s. The inset shows the fluorescence change, $\Delta F / F$, at the peak of an observed calcium event for a rectangular region around and including one of the ROls. (d) $\Delta F / F$ time traces of the three neurons; the colors of the traces are matched to the colors of the ROIs in (c). The data analysis procedure is described in Supplementary Note 3 in the Supplementary Material. The neuron corresponding to the red arrow in (b) and (c) exhibited higher contrast for light-sheet relative to epi-illumination by a factor of $\approx 3.4 \times$. The fluorescent point source corresponding to the yellow arrow in (a) could not be identified as a neuron due to a lack of timedependent fluorescence; a contrast enhancement of $\approx 2.7 \times$ was observed for light-sheet illumination compared to epi-illumination. The scale bars of (a)-(c) are $50 \mu \mathrm{m}$, and the dashed boxes denote the approximate positions of the shanks in the image planes. The intensity grayscales in (a) and (b) are set by the maximum and minimum pixel values outside the dashed boxes, and the grayscale of (c) is set by 99th and 1st percentiles of pixel intensities. We previously reported this data in Ref. 26. 
somatosensory cortex. Time-dependent fluorescence using probe-illumination was observed with a maximum $\Delta F / F$ of 0.12 , and contrast enhancements were observed compared to epi-illumination. In this case, it proved difficult to establish statistical comparisons due to the low number of neurons exhibiting activity in the anesthetized mouse. The probe used for this test was an earlier prototype, which pre-dated our foundry-fabricated probes; details of these earlier devices are listed in Supplementary Note 4 in the Supplementary Material. Compared to the foundry-fabricated probes, the earlier prototype probes were fabricated on a smaller (100-mm diameter) wafer and had thinner shanks ( $\approx 18 \mu \mathrm{m}$ thickness); all other probe dimensions were not significantly different. The photonic circuitry design and the optical functionality were nearly identical between these two types of probes, and thus, the foundry-fabricated probes are expected to also be compatible with in vivo imaging, albeit with additional tissue displacement. The shank thickness of the foundry-fabricated probes can be reduced through fabrication process optimization.

\section{Discussion and Conclusion}

We have conceived of and demonstrated a new paradigm for implantable photonic neural probes that enables lensless delivery of multiple addressable light sheets. These can facilitate 1P-LSFM at arbitrary depths in mammalian brains and other non-transparent tissues. The light-sheet profiles were experimentally characterized, and the probes were validated by fluorescence imaging in fixed tissue and by functional imaging in vitro. This imaging approach requires no active components on the probe, which can otherwise induce deleterious tissue heating. By contrast, miniaturized forms of digitally scanned 1P- or 2P-LSFM would likely require actuators on the probe or in close proximity thereof. As the light-sheet neural probes are passive, the ultimate volumetric imaging rate is similar to that of conventional light-sheet imaging systems. In our probe-based implementation, it is limited by external components that include the electrically tunable lens, MEMS mirror, and the image sensor. The apparatus we employ here is not yet fully optimized to achieve maximum volumetric imaging rates and is primarily limited by the tunable lens, which has a response time of $25 \mathrm{~ms}$. Other system components are faster; the MEMS mirror step time is $\sim 5 \mathrm{~ms}$, and the maximum full frame rate of the camera is 101 frames per second. Accordingly, with optimized component choices and engineering of the imaging system, volumetric imaging rates $\geq 30$ volumetric scans per second will be attainable. ${ }^{38}$ Although continuouswave light was used in our experiments, future implementations can employ pulsed light to mitigate any potential phototoxicity and photodamage.

The light sheets created by our probes are synthesized from an incoherent sum of multiple GC optical emissions. We expect sheets generated by neural probes or by conventional lightsheet microscopes to be similarly affected by scattering within brain tissue. Optical scattering has been evaluated for conventional light-sheet microscopes in Ref. 39 and for nanophotonic GC emissions in Refs. 27 and 30.

In this first demonstration of photonic neural probe-enabled LSFM, significant image contrast enhancements have been observed relative to epifluorescence imaging. However, the light sheet thicknesses of the neural probes in this work $(<16 \mu \mathrm{m}$ average FWHM over a 300- $\mu \mathrm{m}$ propagation distance in air) are larger than those of conventional light-sheet microscopes, which readily achieve $\sim 5 \mu \mathrm{m}$ FWHM sheet thicknesses over $300 \mu \mathrm{m}$ sheet lengths at a wavelength of $488 \mathrm{~nm} .{ }^{13}$ Therefore, the illumination geometry advantages of these first light-sheet neural probe designs are accompanied by lower attainable image contrasts compared to conventional lightsheet microscopes. Optimization of the GC emitter design may reduce the sheet thickness of the neural probes and correspondingly increase the achievable image contrast. One potential approach is to implement a non-uniform GC design wherein the GC periods are selected to enable focusing ${ }^{40}$ along the thickness-axis of the light sheet.

In future iterations of our probe designs, their photonic circuits can be further optimized by leveraging state-of-the-art integrated photonic technology. For example, the sheet density may be increased by integrating multiple photonic layers. ${ }^{41}$ Also, the optical transmission of the probes can be increased by roughly an order of magnitude with efficient fiber-to-chip edge couplers ${ }^{42}$ and optimized low-loss components; the fiber-to-chip coupling efficiency of the edge couplers in this work was limited to $\approx 14 \%$ with optimal alignment. ${ }^{34}$ Optimized packaging solutions can also mitigate transmission variations among light sheets and improve the thermal stability of the 
packaged probes. (The latter may eliminate the turn-on-transient documented in Fig. 15 in Sec. 5.3.) Optimization of probe transmission and packaging can also minimize potential tissue heating arising from the packaged probes absorbing extraneous scattered light from on-chip photonic circuitry and edge couplers. In addition, implanting multiple light-sheet neural probes into brain tissue is a potential method to increase the illuminated field of view and improve the light sheet uniformity, e.g., by implanting multiple neural probes around the GRIN lens in the proposed imaging configuration in Fig. 2(b). To provide clearance between the probes, the onchip photonic circuitry may require modifications so that the edge couplers and the fiber bundle are in line with the shanks.

With their microscale form factors, ultrathin profiles, and their compatibility with sheetnormal imaging using implantable GRIN lens endoscopes, the light-sheet photonic neural probes we have demonstrated herein can engender exciting and powerful new variants of LSFM, both for deep brain imaging and for behavioral experiments with freely moving animals. Beyond LSFM imaging, these neural probes can also be used for laminar optogenetic neural stimulation, e.g., for addressing individual cortical layers. When combined with a new class of implantable neural probes containing photodetector arrays that is now emerging, ${ }^{43}$ they can enable complex image reconstruction realized by means of a complete, implantable lensless imaging system.

\section{Methods}

\subsection{Foundry Fabrication of Photonic Neural Probes}

Neural probes were fabricated on 200-mm-diameter Si wafers at Advanced Micro Foundry (AMF). First, the $1.48-\mu \mathrm{m}$ and 135 -nm-thick $\mathrm{SiO}_{2}$ bottom cladding and $\mathrm{SiN}$ waveguide layer were deposited by plasma-enhanced chemical vapor deposition (PECVD). Fully etched SiN waveguides were formed using deep ultraviolet (DUV) lithography followed by reactive-ion etching (RIE), and the $1.55-\mu \mathrm{m}$-thick $\mathrm{PECVD} \mathrm{SiO}_{2}$ top cladding layer was then deposited. Deep trenches were etched to define the probe shape and form facets for edge couplers. Finally, as in Ref. 44 , backgrinding was used to thin the wafers to $\approx 50$ to $92 \mu \mathrm{m}$, which exposed the deep trenches and separated the probes on the grinding tape (autodicing). Chemical mechanical planarization (CMP) was used for layer planarization during the fabrication. The fabrication process and waveguide characteristics are described in more detail in Ref. 34.

\subsection{Neural Probe Packaging}

The probe chip was first epoxied to a 3D-printed chip carrier. The image fiber bundle (Fujikura FIGH-06-300S) was connected and aligned to the scanning optical system [Fig. 2(a)]. The fiber bundle was aligned and then UV-epoxied to the probe chip and the chip carrier; the emitted optical power from the probe was monitored during the process. The probe chip (excluding the shanks) and the fiber bundle were then coated with optically opaque epoxy to block stray light not coupled to the on-chip waveguides. The chip carrier had a steel rod attached to the proximal end, and this steel rod was connected to additional rods to mount the packaged probe on a 4-axis micro-manipulator (QUAD, Sutter Instrument Company, Novato, California).

\subsection{Spatial Addressing of the Neural Probes}

The 2-axis MEMS mirror in the external scanning optical system, Fig. 2(a), had a nominal maximum mechanical tilt angle of $\pm 5.5 \mathrm{deg}$ and a mirror diameter of $3.6 \mathrm{~mm}$ (A7B2.1-3600AL, Mirrorcle Technologies Inc., Richmond, California). The scanning system used bi-convex lenses with 35- and 150-mm focal lengths and a 20× objective lens (Plan Apochromat, 20-mm working distance, 0.42 numerical aperture, Mitutoyo Corporation, Kawasaki, Japan). The loss of the scanning system (from the input laser beam to the distal facet of the image bundle) was typically $40 \%$ to $60 \%$. The 488-nm laser (OBIS $488 \mathrm{~nm}$ LS $150 \mathrm{~mW}$, Coherent Inc., Santa Clara, California) was fiber-coupled to a single-mode fiber (460-HP, Nufern Inc., East Granby, 
Connecticut), which was connected to a fiber collimator that formed the free-space laser beam input to the scanning system. The input beam was gated by a mechanical shutter. The input polarization to the scanning system was set via an inline fiber polarization controller.

\subsection{Fluorescein Beam Profiling}

The neural probes were dipped into $10 \mu$ mol fluorescein solutions ( $\mathrm{pH}>9$ ), Fig. 3(a). Topdown sheet profiles were measured using an epifluorescence microscope above the probe. Side profiles showing the sheet thicknesses were measured using an additional microscope positioned at the side of the chamber containing the fluorescein. One of the walls of the chamber was removed and replaced with a coverslip to create a viewing port with low optical distortion for the side microscope. Bandpass optical filters on both microscopes rejected excitation light from the probe and transmitted the emission light from the fluorescein. The insertion axis of the micromanipulator holding the probe was angled such that the sheets propagated parallel to the surface of the fluorescein solution with the probe immersed.

\subsection{Free-Space Beam Profiling}

Coverslips with a fluorescent thin film were fabricated by mixing fluorescein (free acid) powder with SU-8 photoresist ${ }^{45}$ and spin coating it onto $\approx 170-\mu$ m-thick coverslips. After curing, an $\approx 8-\mu$ m-thick fluorescent thin film was formed on one side of the coverslips, which were then cleaved in half to prevent the edge bead from limiting the probe-to-coverslip distance. The coverslip was fixed above the probe with the shanks, coverslip, and optical table parallel (Fig. 4). The fluorescent film was on the bottom side of the coverslip, closest to the probe. An epifluorescence microscope above the coverslip imaged the fluorescent patterns created by the intersection of the probe light sheets and the thin film. Vertical translation of the probe enabled volumetric profiling of each sheet for measurements of the sheet thickness and propagation angle. The sheet propagation angles were used to convert the micro-manipulator vertical translation step size into sheet propagation distance step sizes, and the angles were also used to calculate the sheet thicknesses from the angled projections on the coverslip. To verify the uniformity and linearity of the thin film's fluorescence, volumetric profiles were captured over input optical powers spanning roughly an order of magnitude and at multiple positions on the coverslip. Measured average sheet thicknesses varied by $<2 \mu \mathrm{m}$ throughout the trials.

\subsection{Fluorescent Beads in Agarose}

To prepare the agarose blocks with fluorescent beads, $100 \mathrm{mg}$ of agarose powder was mixed with $10 \mathrm{ml}$ of Milli-Q water to form a 1\% agarose solution. The solution was heated until boiled, and after cooling, $50 \mu \mathrm{l}$ of yellow-green fluorescent microbeads (3- $\mu \mathrm{m}$ bead diameter, $2.5 \%$ concentration, Magsphere, Pasadena, California) were mixed into the solution. The solution was placed on a rocker to evenly distribute the beads, and then, poured into a plastic mold and stored in a refrigerator until solidified. The intensity scales of the grayscale images of fluorescent beads in Fig. 5(d) were set with the bottom and top $1 \%$ of all pixel intensities saturated.

\subsection{Imaging Apparatus}

The fluorescence imaging apparatus, Figs. 5(a) and 5(b), includes an epifluorescence microscope (Eclipse FN1, Nikon, Tokyo, Japan) with an sCMOS camera (Zyla 4.2 PLUS, Andor Technology Ltd., Belfast, UK) and an EGFP filter cube (49002, Chroma Technology Corporation, Bellows Falls, Vermont). A 10× objective lens (Mitutoyo Plan Apochromat, 34-mm working distance, 0.28 numerical aperture) was used for the beam characterization and in vitro Cal-520 AM brain slice imaging. A 20× objective (Mitutoyo Plan Apochromat, 20-mm working distance, 0.42 numerical aperture) was used for the fluorescent beads, fixed tissue, and in vitro GCaMP6s brain slice imaging. An electrically tunable lens (Optotune, Dietikon, Switzerland) attached to the back of the objective was used for fast focus adjustments in the fluorescence imaging experiments but not for the beam characterization. The fluorescent beads, fixed tissue, and in vitro imaging used 200-, 500-, and 100-ms camera exposure times, 
respectively. The packaged probe was attached to a 4-axis micromanipulator for positioning the probe in the characterization and imaging experiments. The shanks were aligned to the insertion axis of the micro-manipulator, and in the imaging experiments, the insertion angle was selected for sheet-normal imaging. Since the fiber bundle was not polarization-maintaining and the probe was polarization-sensitive, the bundle was fixed in position during imaging experiments to minimize polarization fluctuations.

\subsection{Animals}

All experimental procedures described here were reviewed and approved by the animal care committees of the University Health Network in accordance with the guidelines of the Canadian Council on Animal Care. Adult Thy1-GCaMP6s mice ${ }^{2}$ (The Jackson Laboratory, Bar Harbor, Maine, stock number 025776) and C57BL/6 mice (Charles River Laboratories, Wilmington, Massachusetts) were kept in a vivarium maintained at $22^{\circ} \mathrm{C}$ with 12 -h light on/off cycle. Food and water were available ad libitum.

\subsection{Fixed Tissue Preparation}

Fixed tissue was prepared from a Thy1-GCaMP6s mouse, postnatal day 172, as $\approx 1$-mmthick transverse slices from the hippocampus (dentate gyrus). Briefly, the animal was anesthetized via an intra-peritoneal injection of sodium pentobarbital $(75 \mathrm{mg} / \mathrm{kg}$, Somnotol, WTC Pharmaceuticals, Cambridge, Ontario, Canada) and transcardially perfused with $1 \times$ phosphate-buffered saline (PBS) followed with paraformaldehyde (PFA) (4\%). Then the extracted brain was kept in PFA at $4^{\circ} \mathrm{C}$ for $12 \mathrm{~h}$. After fixation, the hippocampal slices were prepared in 1× PBS with a vibratome (VT1200S, Leica Biosystems, Wetzlar, Germany).

\subsection{In Vitro Imaging Brain Slice Preparation}

Brain slices were prepared from 30 to 60 day old Thy1-GCaMP6s and C57BL/6 mice for the in vitro GCaMP6s and calcium dye imaging experiments, respectively. The animals were anesthetized with an intra-peritoneal injection of sodium pentobarbital $(75 \mathrm{mg} / \mathrm{kg})$ and transcardially perfused with cold $\left(4^{\circ} \mathrm{C}\right) \mathrm{N}$-methyl-D-glucamine (NMDG) recovery solution ${ }^{46}$ prior to decapitation. The brain was quickly dissected, brain tissues were glued on a vibratome stage, and $450-\mu \mathrm{m}$-thick slices were prepared with the vibratome using iced NMDG solution. The brain slices were then stabilized in NMDG solution at $34^{\circ} \mathrm{C}$ for 12 min while being aerated with carbogen $\left(95 \% \mathrm{O}_{2}, 5 \% \mathrm{CO}_{2}\right)$. Only for experiments with the Cal-520 AM calcium dye, following a 12-min recovery period, slices were rinsed and then bathed in a Cal-520 AM solution (AAT Bioquest, Sunnyvale, California) for 60 to $90 \mathrm{~min}$ at $37^{\circ} \mathrm{C}$. For all in vitro experiments, the slices were then maintained in room temperature incubation solution ${ }^{46}$ for 1 to $8 \mathrm{~h}$ prior to imaging. The Cal-520 AM solution was aerated with carbogen and consisted of $50 \mu \mathrm{g}$ of Cal-520 AM mixed with $20 \mu \mathrm{l}$ of $20 \%$ Pluronic F-127 in dimethyl sulfoxide (DMSO) (Sigma-Aldrich, St. Louis, Missouri) and then diluted in 4 to $6 \mathrm{ml}$ of incubation solution to a final concentration of 7 to $10 \mu \mathrm{Mol}$. During imaging of a slice, the slice was mounted in a perfusion chamber with a constant flow of rodent artificial cerebrospinal fluid (aCSF) solution ${ }^{46}$ aerated with carbogen. A $100-$ to $200-\mu \mathrm{Mol}$ solution of 4-aminopyridine (4-AP) was added to the aCSF bath to put the neurons in a hyperexcitable state for increased neuronal activity. ${ }^{37}$ For the Thy1-GCaMP6s imaging, a transverse slice prominently featuring the auditory cortex was chosen, and for the Cal-520 AM imaging, a sagittal slice prominently featuring the cerebellum was chosen. The cerebellum was chosen since we observed that it had high neuron activity density. The cerebellum could not be chosen for the Thy1-GCaMP6s experiment due to the low labeling density in the cerebellum for this strain. ${ }^{2}$

\subsection{In Vivo Imaging}

First, the Thy1-GCaMP6s mouse (postnatal day 47) was anesthetized, by induction with 5\% and maintenance with $1 \%$ to $2 \%$ isoflurane/oxygen anesthetic, and secured in a stereotaxic frame via earbars (Model 902, David Kopf Instrument, Tujunga, California). A craniotomy was performed 
over the parieto-temporal lobe with an electric drill by removing a square portion of the skull posterior to the bregma and anterior to the lambda bony landmarks (1-mm lateral to midline and 1-mm medial to the superior attachment of the temporalis muscle). The dura was gently removed with a 30 gauge needle for higher image quality and easier probe insertion. Once the cranial opening was complete, the stereotaxic frame-with the mouse secured-was moved under the epifluorescence microscope and the brain surface was irrigated with saline. Initial epifluorescence brain imaging was performed to identify the somatosensory cortex, and an area with few cortical blood vessels in the parieto-temporal lobe at the approximate location of the somatosensory cortex was chosen for probe insertion. Next, the probe was slowly inserted into the brain to a maximum depth of $200 \mu \mathrm{m}$ via the micro-manipulator, and then, fluorescence imaging was performed using both probe- and epi-illumination. The probe insertion angle was set so that the light sheet was roughly parallel to the surface of the brain. The in vivo fluorescence imaging used a 200-ms exposure time and $2 \times 2$ binning; binning was not used in the other imaging experiments in this work.

\section{Appendices}

\subsection{Appendix A: Light-Sheet and Epi-Illumination Intensity Estimates}

For the fixed tissue fluorescence imaging in Fig. 6, the epi-illumination intensity at the surface of the brain slice was measured to be approximately $0.5 \mathrm{~mW} / \mathrm{mm}^{2}$. The Sheets 3 and 4 output

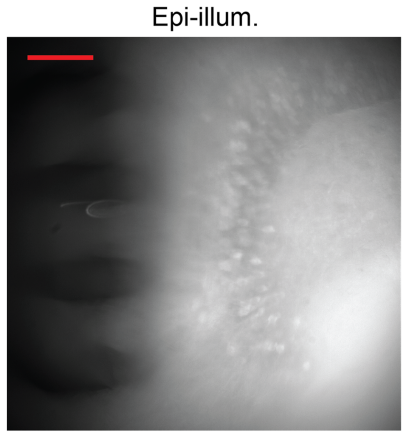

(a)

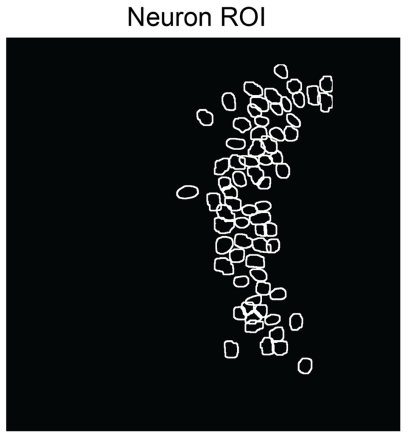

(c)

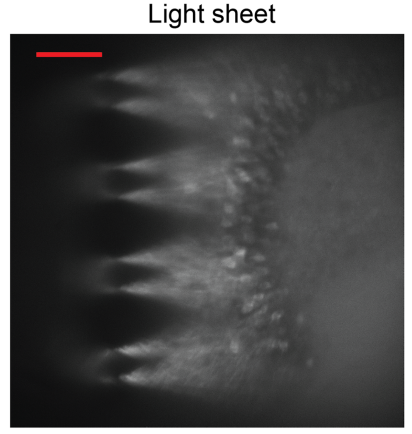

(b)

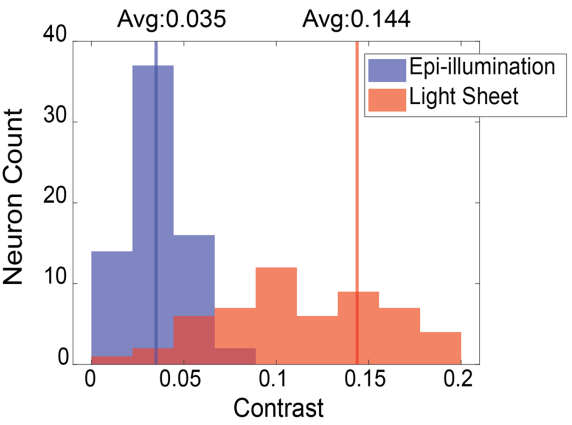

(d)

Fig. 10 Additional data for the Thy1-GCaMP6s fixed mouse brain tissue imaging in Fig. 6(a). Microscope epi-illumination and light-sheet neural probe illumination (Sheet 3, Probe 1) are compared using a reduced light-sheet intensity relative to Fig. 6(a). Top-down fluorescence images of (a) epi-illumination and (b) light-sheet illumination. The epi-illumination image data in (a) are the same as in Fig. 6(a). The scale bars are $100 \mu \mathrm{m}$. (c) ROls of identified neurons. (d) Histograms of image contrast for the identified neurons. The light-sheet image was taken during the turn-off transient of the shutter gating the input laser beam to the scanning system, and therefore, the light-sheet image is an average of multiple intensities over the camera exposure time (500 ms). The average intensity of the light-sheet image is $2.85 \times$ lower than that of Fig. $6(\mathrm{a})$. The light-sheet average neuron contrast in (d) was not significantly changed compared to the higher sheet intensity image in Fig. 6(a). 
powers from the neural probe were approximately 60 and $3 \mu \mathrm{W}$, respectively. Neglecting tissue scattering, at a propagation distance of $200 \mu \mathrm{m}$ from the probe, assuming the sheet width and thickness from Figs. 3(b) and 4(b), respectively, yields Sheets 3 and 4 intensity estimates of $\approx 11$ and $0.6 \mathrm{~mW} / \mathrm{mm}^{2}$, respectively.

For the GCaMP6s and Cal-520 AM in vitro imaging in Figs. 7, 8, 12, and 13, the epiillumination intensities were measured to be approximately 0.3 and $1.0 \mathrm{~mW} / \mathrm{mm}^{2}$, respectively. For light-sheet illumination, neglecting tissue scattering and assuming the sheet width and thickness from Figs. 3(c) and 4(b), at a propagation distance of $200 \mu \mathrm{m}$ from the probe, the light sheet intensity estimate in these experiments is $13 \mathrm{~mW} / \mathrm{mm}^{2}$.

Tissue scattering is expected to significantly reduce the intensity of the light-sheet and epi-illumination. Rather than quantifying this effect and matching the intensities inside the tissue for comparing light-sheet and epi-illumination, we have varied the illumination intensity and confirmed no large changes in the contrast enhancement of light-sheet probe illumination relative to epi-illumination. Figure 10 shows fixed tissue imaging with reduced light-sheet illumination intensity (relative to Fig. 6) and no significant degradation in the ratio of light-sheet to epi-illumination image contrast. In Fig. 11, the epi-illumination intensity is varied over a $\approx 4 \times$ range for the Thy1-GCaMP6s mouse brain slice imaged in Fig. 7. For the two neurons analyzed, the image contrast does not continuously increase with epiillumination intensity and remains significantly less than that of light-sheet illumination in all cases.

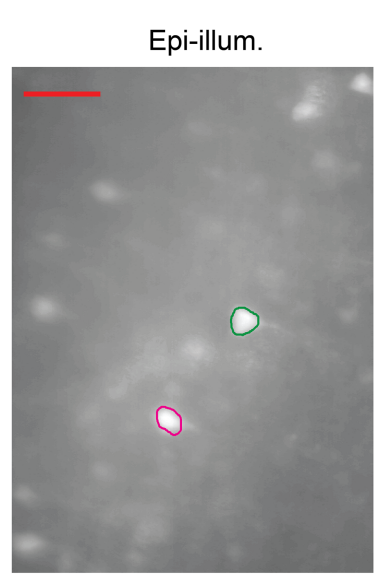

(a)

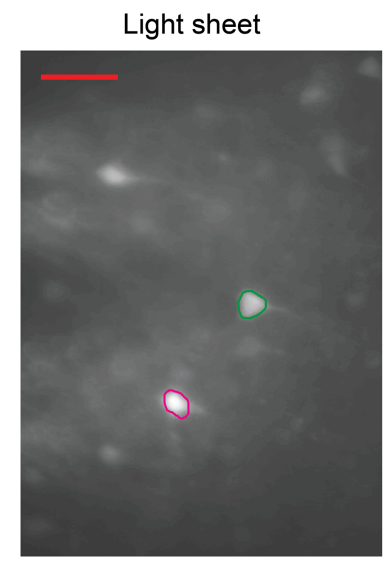

(b)

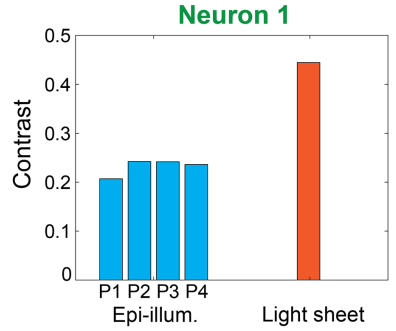

Neuron 2

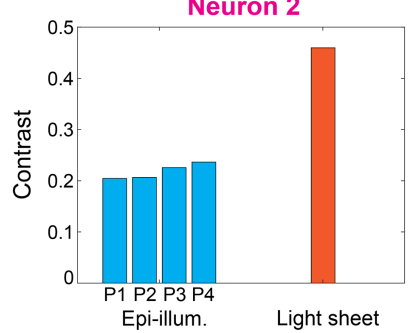

(c)

Fig. 11 Image contrast comparison of two neurons in the Thy1-GCaMP6s brain slice imaged in Fig. 7 at multiple microscope epi-illumination intensities and with a single light-sheet probe illumination intensity (Sheet 5, Probe 2). Brain slice fluorescence images with (a) epi-illumination and (b) light-sheet probe illumination; the ROls of the two neurons are indicated, and the scale bars are $50 \mu \mathrm{m}$. (c) Image contrast of the neurons at four epi-illumination intensities, P1 to P4, and with light-sheet illumination using the intensity in Fig. 7. The estimated epi-illumination intensities (P1, P2, P3, and P4) were $0.7,1.1,1.9$, and $2.8 \mathrm{~mW} / \mathrm{mm}^{2}$, respectively, and the corresponding contrasts span $20.7 \%$ to $24.2 \%$ for neuron 1 and $20.5 \%$ to $23.6 \%$ for neuron 2 . The epi-illumination neuron contrasts are consistently lower than the light-sheet contrasts. The neuron image contrast is calculated using Eq. (1) in Supplementary Note 1 in the Supplementary Material, averaging over 50 to $107 \mathrm{~s}$ of video. The two neurons were selected for their ease of identification in both epi- and light-sheet illumination images. At each illumination condition, over the time averaging window, the time-varying neuron intensity averaged over the ROI had a standard deviation relative to the median of $2.7 \%$ to $4.5 \%$ for epi-illumination and $11.9 \%$ to $12.8 \%$ for light-sheet illumination. 

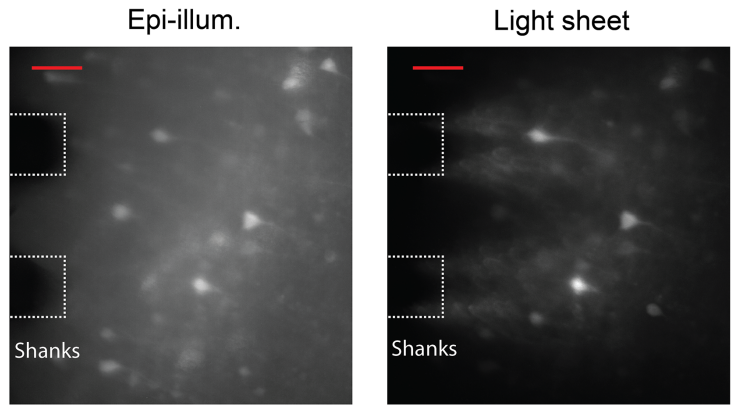

(a)

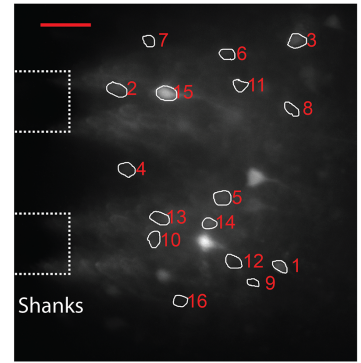

(b)

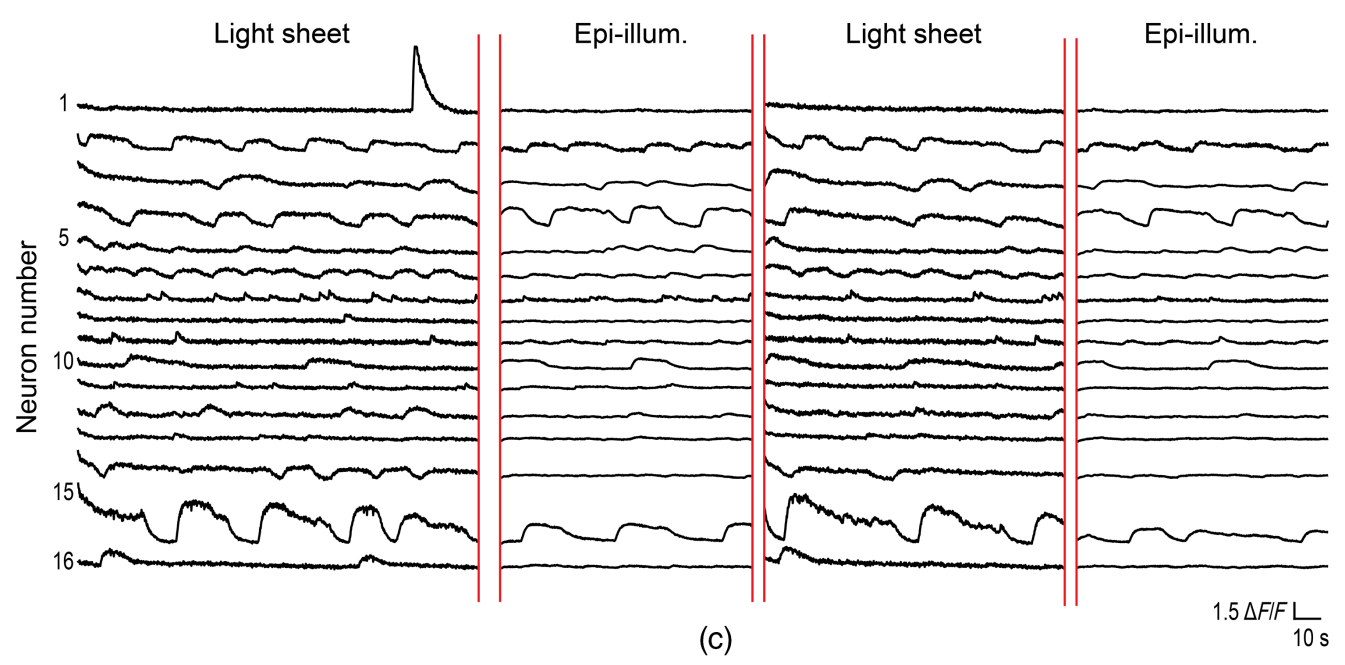

Fig. 12 Additional data for the in vitro Thy1-GCaMP6s mouse brain slice calcium imaging in Fig. 7 showing the complete time traces as the illumination was switched between light-sheet and epi-illumination. (a) Maximum projection images of the first segments of the recorded video for light-sheet probe illumination and microscope epi-illumination. The scale bars are $50 \mu \mathrm{m}$. (b) Lightsheet maximum projection image from (a) with ROls for identified neurons shown; (a) and (b) are repeated from Fig. 7 to show the neuron ROls for the time traces in (c). (c) Fluorescence change, $\Delta F / F$, time traces of the neurons identified in (b) as the illumination was switched between lightsheet and epi-illumination. Gaps in the time traces correspond to periods when no illumination was applied. The time traces correspond to the video used to generate the neuron contrast box plots in Fig. 7(d).

\subsection{Appendix B: Additional in Vitro Imaging Data}

Figures 12 and 13 show the complete time traces for the GCaMP6s and Cal-520 AM in vitro brain slice imaging experiments in Figs. 7 and 8, respectively, as the illumination was switched between light-sheet and epi-illumination. Figure 14 shows additional Cal-520 AM in vitro brain slice imaging data with light-sheet neural probe illumination; the brain slice is the same as Figs. 8 and 13 , but the probe was inserted at a different position in the slice.

\subsection{Appendix C: Turn-on Transient}

The packaged light-sheet neural probes exhibited a turn-on transient upon applying the optical input. Figure 15 shows an example of this turn-on transient during fluorescence imaging of a Thy1-GCaMP6s mouse brain slice. The image intensity in the illuminated area decreased by $\approx 20 \%$ to $25 \%$ in the first $5 \mathrm{~s}$ of illumination, and then continued to decrease at a slower rate. This turn-on transient may be due to thermal expansion of the probe packaging upon absorbing stray input light not coupled to the probe chip. The measured intensity time traces in Fig. 15 are a combination of the turn-on transient in probe illumination and any photobleaching of the brain slice that occurred. 


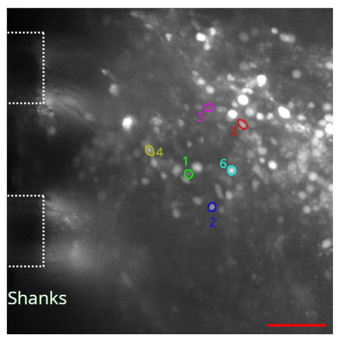

(a)

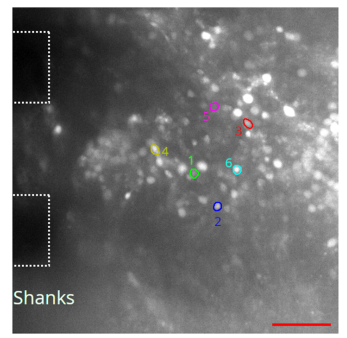

(b)

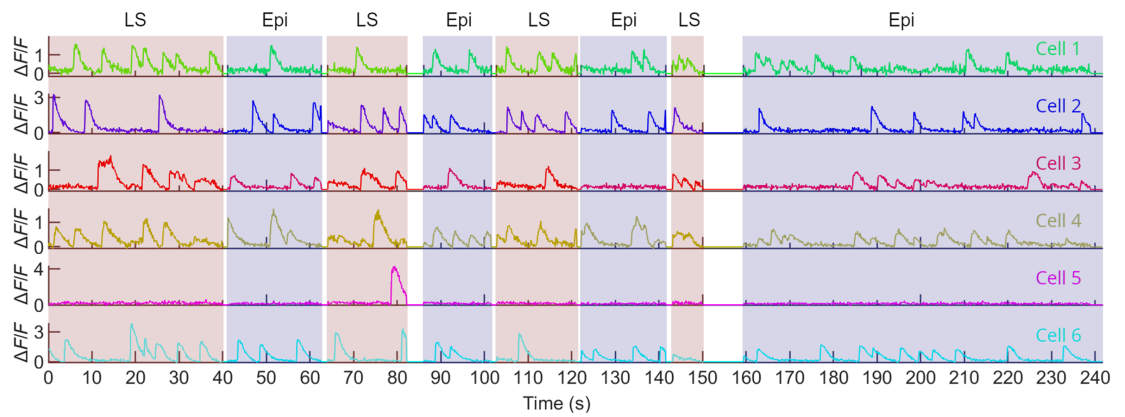

(c)

Fig. 13 Additional data for the in vitro Cal-520 AM loaded mouse brain slice calcium imaging in Fig. 8. Maximum projection fluorescence images of segments of the recorded video for (a) lightsheet and (b) epi-illumination. Annotations show the approximate positions of the shanks in the image plane, and the scale bars are $50 \mu \mathrm{m}$. The ROls of the six cells selected for time traces in Fig. 8 are shown. (c) $\Delta F / F$ time traces of the six cells identified in (a) and (b) as the illumination was switched between light-sheet ("LS") and epi-illumination (“Epi"). The colors and numbers of the cell ROls correspond to the time traces. Gaps in the time traces correspond to periods when no illumination was applied. The maximum projection images in (a) and (b) are taken over the first light-sheet and epi-illumination time segments in (c).

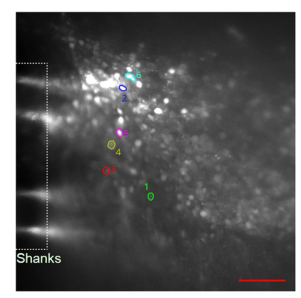

(a)

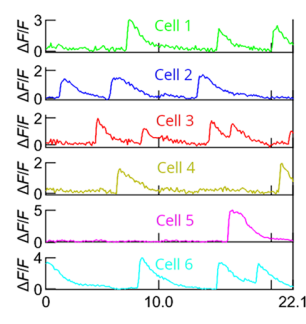

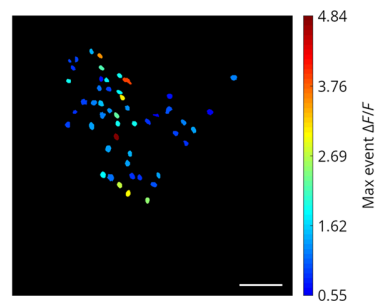

(c) (b)

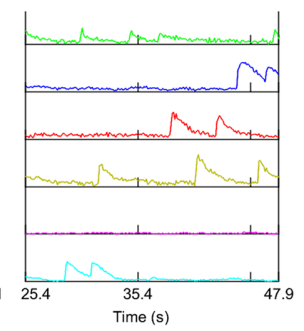

(d)

Fig. 14 Additional in vitro calcium imaging of a Cal-520 AM loaded mouse brain slice using lightsheet probe illumination from Probe 2. The brain slice is the same as Figs. 8 and 13 , but the probe was inserted at a different position in the slice. (a) Maximum projection fluorescence image of a segment of the recorded video with annotations to show the approximate position of the shanks in the image plane. (b) Number of calcium events and (c) maximum $\Delta F / F$ observed for all identified cells; (b) and (c) are of the same scale as (a), and the scale bars are $50 \mu \mathrm{m}$. (d) $\Delta F / F$ time traces of six cells. The number and color-coded ROls for these cells are shown in (a). Breaks in the time traces correspond to periods when the illumination was switched to epi-illumination. The maximum projection image in (a) is taken over the first time segment in (d). 


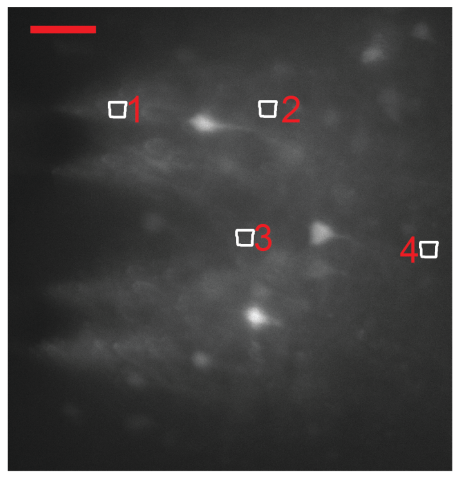

(a)

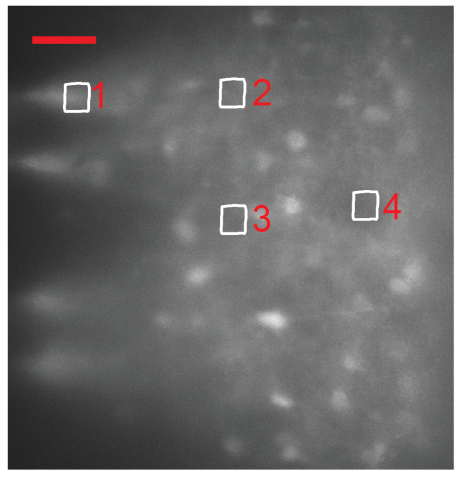

(c)

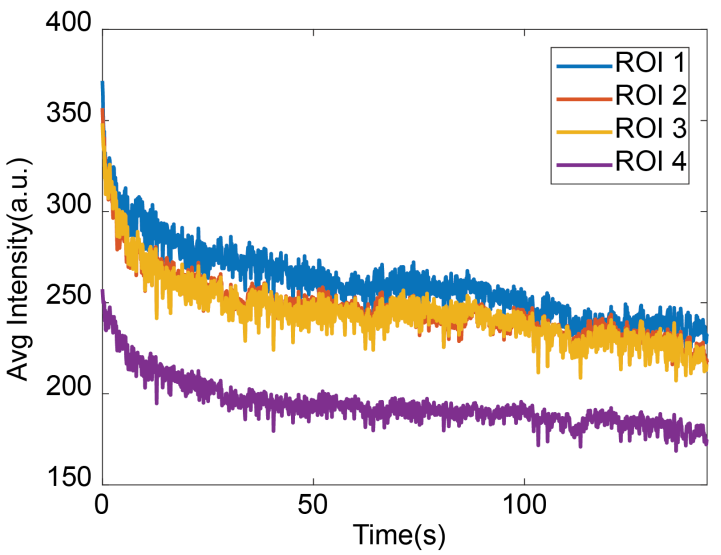

(b)

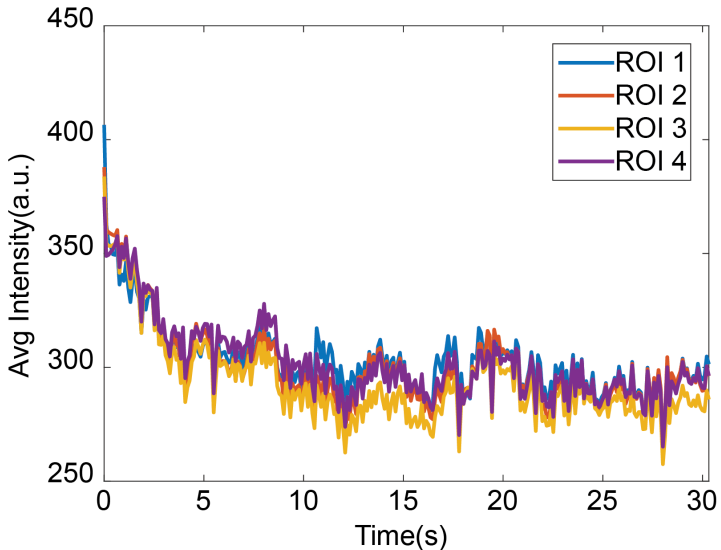

(d)

Fig. 15 Example light-sheet neural probe (Probe 2) fluorescence image intensity turn-on transients for in vitro Thy1-GCaMP6s mouse brain slice calcium imaging. (a) and (c) Fluorescence images of two brain slices from the same mouse with four ROIs labeled in each. The ROls are outside the observable neurons and were selected to sample the neuropil fluorescence at different positions in the sheet. (b) and (d) Image intensity time traces averaged over each ROI; (b) corresponds to the imaging in (a), and (d) corresponds to (c). The beginning of the time-axis corresponds to the time at which the light-sheet illumination was turned on. The scale bars in (a) and (c) are $50 \mu \mathrm{m}$. The data in (a) and (b) are part of the same imaging experiment presented in Fig. 7.

\subsection{Appendix D: Supplementary Videos}

Four supplementary videos are included in this work, and descriptions are as follows.

Video S1 Top-down microscope imaging of Probe 3 with switching between light sheets. The video is real-time (Video S1, 1.96 MB, MP4 [URL: https://doi.org/10.1117/1.NPh.8.2 .025003.4]).

Video S2 Side microscope fluorescence imaging of Probe 1 immersed in a fluorescein solution with switching between the five light sheets. The power of the sheets was roughly equalized here by not optimally aligning the MEMS mirror for the sheets with relatively high transmission. Additional ambient illumination was applied to make the shanks visible. The video is real-time (Video S2, 17.4 MB, MP4 [URL: https://doi.org/10.1117/1.NPh.8.2 .025003.5]).).

Video S3 Fluorescence imaging of 3- $\mu \mathrm{m}$-diameter fluorescent beads suspended in an agarose block with light-sheet illumination from Probe 1 and imaging using the epifluorescence microscope above the sample. At about $17 \mathrm{~s}$, the illumination is switched from light-sheet probe illumination to epi-illumination from the microscope. During light-sheet probe illumination, switching between Sheets 1, 3, and 5 is performed. The tunable lens is synchronized to the 
sheet switching to focus the collection optics on the depth planes corresponding to each sheet. After switching to epi-illumination, the tunable lens focus switching continues and shows epifluorescence imaging of the same depth planes imaged with light-sheet probe illumination. The video is real-time (Video S3, 12.9 MB, MP4 [URL: https://doi.org/10.1117/1.NPh.8.2 .025003.6]).).

Video S4 In vitro calcium imaging with epi-illumination of a Cal-520 AM loaded brain slice from a wild type mouse. This is a sample of the calcium imaging video corresponding to Fig. 13. The video is from the same experiment as Video 3 but with epi-illumination instead of light-sheet probe illumination. The video is real-time (Video S4, 13.9 MB, MP4 [URL: https://doi.org/10 .1117/1.NPh.8.2.025003.7]).).

\section{Disclosures}

The authors declare no competing interests.

\section{Acknowledgments}

This work was supported by the National Institutes of Health, Awards NS090596 and NS099726; Canadian Institute of Health Research, Award FRN151949; and the Natural Sciences and Engineering Research Council of Canada, Award CHRPJ 508406. Funding support from the Canadian Foundation for Innovation and Ontario Research Fund is also gratefully acknowledged. W.D.S. was supported by the Kavli Nanoscience Institute Prize Postdoctoral Fellowship in Applied Physics and Materials Science. A.F. was supported by the Clinician Investigator Program, University of Manitoba. The authors thank Michael Chang and Azadeh Naderian at the Krembil Research Institute for their assistance with the animal colonies and genotyping. The authors also thank Alex Jacob in the group of Professor Sheena Josselyn at SickKids Research Institute (Toronto, Canada) for his advice on GCaMP6 functional imaging.

\section{Code, Data, and Materials Availability}

The data are available from the corresponding authors upon reasonable request.

\section{References}

1. K. Deisseroth, "Optogenetics: 10 years of microbial opsins in neuroscience," Nat. Neurosci. 18, 1213-1225 (2015).

2. H. Dana et al., "Thy1-GCaMP6 transgenic mice for neuronal population imaging in vivo," PLoS One 9, e108697 (2014).

3. M. Inoue et al., "Rational engineering of XCaMPs, a multicolor GECI suite for in vivo imaging of complex brain circuit dynamics," Cell 177, 1346-1360 (2019).

4. K. K. Ghosh et al., "Miniaturized integration of a fluorescence microscope," Nat. Methods 8, 871-878 (2011).

5. D. G. Ouzounov et al., "In vivo three-photon imaging of activity of GCaMP6-labeled neurons deep in intact mouse brain," Nat. Methods 14, 388-390 (2017).

6. M. B. Bouchard et al., "Swept confocally-aligned planar excitation (SCAPE) microscopy for high speed volumetric imaging of behaving organisms," Nat. Photonics 9, 113-119 (2015).

7. A. M. Packer et al., "Simultaneous all-optical manipulation and recording of neural circuit activity with cellular resolution in vivo," Nat. Methods 12, 140-146 (2015).

8. J. H. Marshel et al., "Cortical layer-specific critical dynamics triggering perception," Science 365(6453), eaaw5202 (2019).

9. C. A. Combs, "Fluorescence microscopy: a concise guide to current imaging methods," Curr. Protoc. Neurosci. 50, 2.1.1-2.1.25 (2010).

10. W. Denk, J. Strickler, and W. Webb, "Two-photon laser scanning fluorescence microscopy," Science 248, 73-76 (1990). 
11. C. Tischbirek et al., "Deep two-photon brain imaging with a red-shifted fluorometric $\mathrm{Ca}^{2+}$ indicator," Proc. Natl. Acad. Sci. USA 112, 11377-11382 (2015).

12. S. R. Schultz et al., "Advances in two photon scanning and scanless microscopy technologies for functional neural circuit imaging," Proc. IEEE 105, 139-157 (2017).

13. E. M. Hillman et al., "Light-sheet microscopy in neuroscience," Аnnи. Rev. Neurosci. 42, 295-313 (2019).

14. P. J. Keller et al., "Reconstruction of zebrafish early embryonic development by scanned light sheet microscopy," Science 322, 1065-1069 (2008).

15. J. Mertz, "Optical sectioning microscopy with planar or structured illumination," Nat. Methods 8, 811-819 (2011).

16. R. M. Power and J. Huisken, "A guide to light-sheet fluorescence microscopy for multiscale imaging," Nat. Methods 14, 360-373 (2017).

17. P. J. Keller and M. B. Ahrens, "Visualizing whole-brain activity and development at the single-cell level using light-sheet microscopy," Neuron 85, 462-483 (2015).

18. P. Haslehurst et al., "Fast volume-scanning light sheet microscopy reveals transient neuronal events," Biomed. Opt. Express 9, 2154-2167 (2018).

19. V. Voleti et al., "Real-time volumetric microscopy of in vivo dynamics and large-scale samples with SCAPE 2.0," Nat. Methods 16, 1054-1062 (2019).

20. W. Zong et al., "Fast high-resolution miniature two-photon microscopy for brain imaging in freely behaving mice," Nat. Methods 14, 713-719 (2017).

21. A. D. Jacob et al., "A compact head-mounted endoscope for in vivo calcium imaging in freely behaving mice," Curr. Protoc. Neurosci. 84, e51 (2018).

22. A. de Groot et al., "NINscope, a versatile miniscope for multi-region circuit investigations," eLife 9, e49987 (2020).

23. T. Shuman et al., "Breakdown of spatial coding and interneuron synchronization in epileptic mice," Nat. Neurosci. 23, 229-238 (2020).

24. C. J. Engelbrecht, F. Voigt, and F. Helmchen, "Miniaturized selective plane illumination microscopy for high-contrast in vivo fluorescence imaging," Opt. Lett. 35, 1413-1415 (2010).

25. F. Ye, B. W. Avants, and J. T. Robinson, "Light sheet illumination with an integrated photonic probe," in Conf. Lasers and Electro-Opt., JW2A.142, Optical Society of America (2016).

26. W. D. Sacher et al., "Nanophotonic neural probes for in vivo light sheet imaging," in Conf. Lasers and Electro-Opt., SM4H.6, Optical Society of America (2019).

27. E. Segev et al., "Patterned photostimulation via visible-wavelength photonic probes for deep brain optogenetics," Neurophotonics 4, 011002 (2016).

28. W. D. Sacher et al., "Beam-steering nanophotonic phased-array neural probes," in Conf. Lasers and Electro-Opt., ATh4I.4, Optical Society of America (2019).

29. E. Shim et al., "Multisite silicon neural probes with integrated silicon nitride waveguides and gratings for optogenetic applications," Sci. Rep. 6, 22693 (2016).

30. S. Libbrecht et al., "Proximal and distal modulation of neural activity by spatially confined optogenetic activation with an integrated high-density optoelectrode," J. Neurophysiol. 120, 149-161 (2018).

31. A. Mohanty et al., "Reconfigurable nanophotonic silicon probes for sub-millisecond deepbrain optical stimulation," Nat. Biomed. Eng. 4, 223-231 (2020).

32. L. B. Soldano and E. C. M. Pennings, "Optical multi-mode interference devices based on self-imaging: principles and applications," J. Lightwave Technol. 13, 615-627 (1995).

33. H. Chen and A. W. Poon, "Low-loss multimode-interference-based crossings for silicon wire waveguides," IEEE Photonics Technol. Lett. 18, 2260-2262 (2006).

34. W. D. Sacher et al., "Visible-light silicon nitride waveguide devices and implantable neurophotonic probes on thinned $200 \mathrm{~mm}$ silicon wafers," Opt. Express 27, 37400-37418 (2019).

35. A. N. Zorzos et al., "Three-dimensional multiwaveguide probe array for light delivery to distributed brain circuits," Opt. Lett. 37, 4841-4843 (2012).

36. U. Hofmann, J. Janes, and H.-J. Quenzer, "High-Q MEMS resonators for laser beam scanning displays," Micromachines 3, 509-528 (2012).

37. M. Chang et al., "Brief activation of GABAergic interneurons initiates the transition to ictal events through post-inhibitory rebound excitation," Neurobiol. Disease 109, 102-116 (2018). 
38. F. O. Fahrbach et al., "Rapid 3D light-sheet microscopy with a tunable lens," Opt. Express 21, 21010-21026 (2013).

39. A. K. Pediredla et al., "Deep imaging in scattering media with selective plane illumination microscopy," J. Biomed. Opt. 21, 126009 (2016).

40. V. Lanzio et al., "High-density electrical and optical probes for neural readout and light focusing in deep brain tissue," J. Micro/Nanolith. MEMS MOEMS 17, 025503 (2018).

41. W. D. Sacher et al., "Monolithically integrated multilayer silicon nitride-on-silicon waveguide platforms for 3-D photonic circuits and devices," Proc. IEEE 106, 2232-2245 (2018).

42. L. Chen et al., "Low-loss and broadband cantilever couplers between standard cleaved fibers and high-index-contrast $\mathrm{Si}_{3} \mathrm{~N}_{4}$ or Si waveguides," IEEE Photonics Technol. Lett. 22 , 1744-1746 (2010).

43. J. Choi et al., "A 512-pixel, 51-kHz-frame-rate, dual-shank, lens-less, filter-less singlephoton avalanche diode CMOS neural imaging probe," IEEE J. Solid-State Circuits 54, 2957-2968 (2019).

44. S. Herwik, O. Paul, and P. Ruther, "Ultrathin silicon chips of arbitrary shape by etching before grinding," J. Microelectromech. Syst. 20, 791-793 (2011).

45. M. P. Lim et al., "Augmenting mask-based lithography with direct laser writing to increase resolution and speed," Opt. Express 26, 7085-7090 (2018).

46. J. T. Ting et al., "A robust ex vivo experimental platform for molecular-genetic dissection of adult human neocortical cell types and circuits," Sci. Rep. 8, 8407 (2018).

47. "Correct nonuniform illumination and analyze foreground objects," https://www.mathworks .com/help/images/correcting-nonuniform-illumination.html (accessed 20 October 2019).

48. J. Schindelin et al., "Fiji: an open-source platform for biological-image analysis," Nat. Methods 9, 676-682 (2012).

49. G. van Rossum, "Python tutorial," Technical report CS-R9526, Centrum voor wiskunde en informatica (CWI), Amsterdam (1995).

50. A. Giovannucci et al., "CaImAn: an open source tool for scalable calcium imaging data analysis," eLife 8, e38173 (2019).

51. G. Barbera et al., "Spatially compact neural clusters in the dorsal striatum encode locomotion relevant information," Neuron 92, 202-213 (2016).

52. L. Pinto and Y. Dan, "Cell-type-specific activity in prefrontal cortex during goal-directed behavior," Neuron 87, 437-450 (2015).

53. H. Dana et al., "High-performance calcium sensors for imaging activity in neuronal populations and microcompartments," Nat. Methods 16, 649-657 (2019).

Wesley D. Sacher received his $\mathrm{PhD}$ in electrical and computer engineering from the University of Toronto in 2015. From 2015 to 2018, he was a postdoctoral scholar at California Institute of Technology. He is a research group leader at the Max Planck Institute of Microstructure Physics. His current research is focused on integrated photonics for visible wavelengths and neurotechnologies for optogenetics and functional imaging.

Fu-Der Chen received his BASc and MASc degrees in electrical and computer engineering from the University of Toronto, Ontario, Canada, in 2017 and 2020, respectively. Currently, he is working toward his $\mathrm{PhD}$ at the University of Toronto. His research interest is to develop new implantable optogenetics tools with integrated photonics technology.

Homeira Moradi-Chameh completed her PhD focused on neuroscience-electrophysiology at the Medical School of Tarbiat Modares University in 2015. She was a postdoctoral scholar at the University Health Network, Canada, under the mentorship of Dr. Taufik Valiante in 2018. She is currently a scientific associate at the Krembil Research Institute, University Health Network, where she focuses on patch clamp electrophysiology, slice cultures, epilepsy models, and managing local and international research collaborations.

Xianshu Luo received his PhD in electrical and computer engineering from HKUST, Hong Kong, in 2010. He joined IME, A*STAR, as research scientist, and was engaged in research on Si-PIC, HOEIC for various applications. He is now with Advanced Micro Foundry as 
co-founder/research director, focusing on the developing of advanced devices/PICs. He has authored/co-authored more than 160 peer-reviewed journal and conferences papers, 4 book chapters, and holds more than 20 patents.

Anton Fomenko is a neurosurgery resident and $\mathrm{PhD}$ student interested in noninvasive forms of modulating brain activity. At the University of Toronto, his graduate work centers on lowintensity focused ultrasound in human and in animal models under the supervision of Professor Andres Lozano at the Institute of Medical Science. His lifelong academic mission is to contribute to the interface of technology and the brain for the betterment of patient health outcomes.

Prajay T. Shah received his BSc degree from the University of Calgary in 2017. He is currently an $\mathrm{MD} / \mathrm{PhD}$ student and Vanier Scholar at the University of Toronto.

Thomas Lordello is an optical engineer at Lumentum, working on LCoS-based ROADMs. He holds an MASc degree from the University of Toronto, where he worked on in-vivo fluorescence imaging of brain activity using nanophotonic neuroprobes.

Xinyu Liu received his Bachelor of Science degree from Nanjing University, China, in 2010. In 2017, he received his $\mathrm{PhD}$ in electrical engineering from Duke University. He worked at California Institute of Technology as a research staff member from 2017 to 2019, and from 2020 to present.

Ilan Felts Almog is an MIT and University of Toronto alumnus currently in the role of systems developer at Perimeter Medical Imaging AI. His graduate research at the University of Toronto was primarily focused on developing the first reported endoscopic full-field swept-source optical coherence tomography system and investigating its application to deep brain stimulation (DBS) electrode implantation surgery navigation, later shifting to novel imaging techniques for neuron investigation.

Youngho Jung is a postdoctoral fellow in the Department of Nanophotonics, Integration, and Neural Technology at Max Planck Institute of Microstructure Physics, Germany. He received his $\mathrm{PhD}$ in electrical engineering from Korea Advanced Institute of Science and Technology (KAIST) in 2017. His current research interests vary across the area of photonics, in particular, micro/nano fabrication and integration techniques for photonic devices and on-chip systems for communication, imaging, and other emerging applications.

Andres M. Lozano is a professor of neurosurgery at the University of Toronto. He is best known for work in the field of DBS. His team has mapped human brain circuits and advanced novel treatments for Parkinson's disease, depression, anorexia, and Alzheimer's disease. He has over 700 publications and received honors including election to the Royal Society of Canada, the Order of Merit of Spain, and is an officer of the Order of Canada.

Patrick Guo-Qiang Lo received his PhD/ECE from UT/Austin/USA. He was with Integrated Device Technology Inc/USA, where he was involved in R\&D in CMOS manufacturing and integration. From 2004 to 2017, he was with IME/Singapore, he served as the deputy executive director, and also as program director of Nanoelectronics/Photonics, TSV, and Emerging Memory. Since 2017, he, as co-founder, has been with AMF/Singapore focusing on silicon photonics' industrialization. He has (co-) authored $>300$ journal and conference publications, and holds $>50$ granted patents. He was a recipient of the IEEE GES award for best paper (2008), Singapore's National Technology Award (2008), and the President's Technology Award (2010).

Taufik A. Valiante is an associate professor at the University of Toronto, Department of Surgery, Division of Neurosurgery. He additionally holds cross-appointments to biomedical engineering, and electrical and computer engineering. He is a scientist at the Krembil Brain Institute, and director of the Surgical Epilepsy Program, at the Toronto Western Hospital. He is the co-director of the Center for Advancing Neurotechnological Innovation to Application (CRANIA), and director of the newly established CRANIA Neuromodulation Institute, University of Toronto. 
Laurent C. Moreaux received his PhD in physics in 2002 from Paris-Sud University on nonlinear optical microscopies with Jerome Mertz. After a postdoc with Gilles Laurent at Caltech in neurophysiology, he joined the CNRS, France, as a permanent researcher. He returned to Caltech in 2009, working as a research scientist for two divisions-biology and physics-where since 2013 he has been developing neurophotonic probe technologies with Michael Roukes designed to stimulate and monitor brain activity.

Joyce K. S. Poon is the managing director at the Max Planck Institute of Microstructure Physics, a professor of electrical and computer engineering at the University of Toronto, and an honorary professor in the Faculty of Electrical Engineering and Computer Science at the Technical University of Berlin. She and her team specialize in integrated photonics on silicon. She is a fellow of the Optical Society and currently serves as a director-at-large.

Michael L. Roukes is the Frank J. Roshek Professor of physics, applied physics, and bioengineering at Caltech. His scientific interests range from quantum measurement to neurotechnology, with a unifying theme of applying complex nanosystems to precision measurements in the physical and life sciences. Among his honors, he is a fellow of the American Physical Society, a NIH Director's Pioneer Award recipient, and was awarded Chevalier (Knight) de l'Ordre des Palmes Académiques by the Republic of France.

Biographies of the other authors are not available. 